\title{
Sensorimotor Expectations Bias Motor Resonance during Observation of Object Lifting: The Causal Role of pSTS
}

\author{
${ }^{\circledR}$ Guy Rens,,${ }^{1,2,3}$ Vonne van Polanen, ${ }^{1,2}$ Alessandro Botta, ${ }^{4}$ Mareike A. Gann, ${ }^{1,2}{ }^{\circledR}$ Jean-Jacques Orban de Xivry, ${ }^{1,2}$ \\ and ${ }^{\circledR}$ Marco Davare ${ }^{5}$ \\ ${ }^{1}$ Movement Control and Neuroplasticity Research Group, Department of Movement Sciences, Biomedical Sciences Group, KU Leuven, Leuven, \\ 3001, Belgium, ${ }^{2} \mathrm{KU}$ Leuven, Leuven Brain Institute, Leuven, 3001, Belgium, ${ }^{3}$ The Brain and Mind Institute, University of Western Ontario, London, \\ N6A 3K7 Ontario, Canada, ${ }^{4}$ Department of Experimental Medicine, Section of Human Physiology, University of Genoa, Genoa, 16132, Italy, and \\ ${ }^{5}$ Department of Clinical Sciences and Centre for Cognitive Neuroscience, College of Health and Life Sciences, Brunel University London, Uxbridge, \\ UB8 3PN, United Kingdom
}

Transcranial magnetic stimulation studies have highlighted that corticospinal excitability is increased during observation of object lifting, an effect termed "motor resonance." This facilitation is driven by movement features indicative of object weight, such as object size or observed movement kinematics. Here, we investigated in 35 humans ( 23 females) how motor resonance is altered when the observer's weight expectations, based on visual information, do not match the actual object weight as revealed by the observed movement kinematics. Our results highlight that motor resonance is not robustly driven by object weight but easily masked by a suppressive mechanism reflecting the correctness of weight expectations. Subsequently, we investigated in 24 humans (14 females) whether this suppressive mechanism was driven by higher-order corti$\mathrm{cal}$ areas. For this, we induced "virtual lesions" to either the posterior superior temporal sulcus (pSTS) or dorsolateral prefrontal cortex (DLPFC) before having participants perform the task. Importantly, virtual lesion of pSTS eradicated this suppressive mechanism and restored object weight-driven motor resonance. In addition, DLPFC virtual lesion eradicated any modulation of motor resonance. This indicates that motor resonance is heavily mediated by top-down inputs from both pSTS and DLPFC. Together, these findings shed new light on the theorized cortical network driving motor resonance. That is, our findings highlight that motor resonance is not only driven by the putative human mirror neuron network consisting of the primary motor and premotor cortices as well as the anterior intraparietal sulcus, but also by top-down input from pSTS and DLPFC.

Key words: action observation; corticospinal excitability; mirror neurons; motor resonance; object lifting; sensorimotor

Significance Statement

Observation of object lifting activates the observer's motor system in a weight-specific fashion: Corticospinal excitability is larger when observing lifts of heavy objects compared with light ones. Interestingly, here we demonstrate that this weightdriven modulation of corticospinal excitability is easily suppressed by the observer's expectations about object weight and that this suppression is mediated by the posterior superior temporal sulcus. Thus, our findings show that modulation of corticospinal excitability during observed object lifting is not robust but easily altered by top-down cognitive processes. Finally, our results also indicate how cortical inputs, originating remotely from motor pathways and processing action observation, overlap with bottom-up motor resonance effects.

Received Nov. 12, 2019; revised Mar. 22, 2020; accepted Mar. 25, 2020.

Author contributions: G.R. and M.D. designed research; G.R., V.v.P., A.B., and M.A.G. performed research; G.R., V.v.P., A.B., J.-J.O.d.X., and M.D. analyzed data; G.R. wrote the first draft of the paper; G.R., V.v.P., A.B., M.A.G., J.-J.O.d.X., and M.D. edited the paper; G.R. wrote the paper.

The authors declare no competing financial interests.

G.R. is a doctoral student funded by a Research Foundation Flanders (FWO) Odysseus Project (Fonds Wetenschappelijk Onderzoek, Belgium; Grant G/0(51/13N) awarded to M.D. V.v.P. was supported by FWO Postdoctoral Fellowship Grant 12X7118N. M.A.G. was supported by FWO Research Foundation Grant G099516N.

Correspondence should be addressed to Guy Rens at grens@uwo.ca.

https://doi.org/10.1523/JNEUROSCI.2672-19.2020

Copyright $\odot 2020$ the authors

\section{Introduction}

Over two decades ago, Fadiga et al. (1995) demonstrated the involvement of the human motor system in action observation: By applying single-pulse transcranial magnetic stimulation (TMS) over the primary motor cortex (M1), they revealed that corticospinal excitability (CSE) was similarly modulated when observing or executing the same action. In line with the mirror neuron theory, they argued that the motor system could be involved in action understanding through a bottom-up mapping ("mirroring") of observed actions onto the cortical areas that are involved in their execution (for review, see 
Rizzolatti et al., 2014). Consequently, action observation-driven modulation of CSE has been termed "motor resonance."

Recently, TMS studies in humans substantiated that motor resonance reflects movement features within observed actions. For example, Alaerts et al. (2010a,b) demonstrated that motor resonance during observation of object lifting is modulated by observed features indicative of object weight, such as intrinsic object properties (e.g., size), muscle contractions, and movement kinematics. Specifically, CSE is increased when observing lifts of heavy compared with light objects. Interestingly, Alaerts et al. (2012) also demonstrated that weight-driven motor resonance is already present during the observed reaching phase, suggesting an underlying predictive mechanism as well.

However, motor resonance does not seem to be robust. For instance, Buckingham et al. (2014) demonstrated, using the sizeweight illusion, that CSE modulation is driven by object size when observing skilled but not erroneous lifts. In addition, Senot et al. (2011) demonstrated that object weight-driven motor resonance is eradicated when objects with identical appearance but different weights are labeled the same. Last, Tidoni et al. (2013) demonstrated that motor resonance is altered by the intentions conveyed by the observed person: CSE is increased when observing deceptive lifts compared with truthful ones. Although the above studies experimentally manipulated the information participants perceived, they could not investigate whether the participants' expectations changed and to which extent this affected CSE modulation.

In the present study, we investigated whether the observer's expectations alter motor resonance by manipulating the experimental context. We asked participants to perform an object-lifting task in turns with an actor. One group performed the task on objects with congruent only size-weight relationship (i.e., bigheavy or small-light objects; congruent objects), whereas the other group lifted both congruent and incongruent objects (i.e., big-light or small-heavy objects). Based on the findings of Alaerts et al. (2010b, 2012), we hypothesized that motor resonance would be driven (1) by the intrinsic object properties (i.e., size) before observed object lift-off and (2) by the movement kinematics (i.e., actual object weight) after observed lift-off. However, our results revealed that, for the group lifting both congruent and incongruent objects, CSE was decreased when observing lifts of congruent objects, regardless of the object's size and weight. In contrast, CSE was increased when observing lifts of incongruent objects, again regardless of size and weight. As such, motor resonance was not driven by size or weight but rather by congruence of the objects' size-weight relationship.

We conducted a second experiment to investigate whether object weight-driven motor resonance during observed lifting was suppressed by top-down inputs to the motor system: Another group of participants performed the same task on the congruent and incongruent objects after receiving a virtual lesion of either the posterior superior temporal sulcus (pSTS) or dorsolateral prefrontal cortex (DLPFC). We opted for these areas considering their involvement in understanding intentions and motor goals [DLPFC: Miller and Cohen (2001), Kilner (2011); pSTS: Nelissen et al. (2011)] and in recognizing action correctness [DLPFC: Pazzaglia et al. (2008); pSTS: Pelphrey et al. (2004)]. Based on evidence that pSTS is reciprocally connected with the anterior intraparietal cortex (AIP) (Nelissen et al., 2011) and DLPFC with the ventral premotor cortex (PMv) (Badre and D'Esposito, 2009), which are considered key nodes for driving motor resonance (Rizzolatti et al., 2014), we hypothesized that virtual lesion of either region would release the "suppression" and restore weight-driven motor resonance.

\section{Materials and Methods}

\section{Participants}

Sixty-eight participants were recruited from the student body of $\mathrm{kU}$ Leuven (Belgium) and divided into four groups. Nine individuals were excluded before participation based on screening for TMS (Rossi et al., 2011) and/or MRI safety (checklist of local hospital: UZ Leuven). For Experiment 1, 18 individuals (12 females; mean age $\pm \mathrm{SEM}=23.78 \pm$ 0.12 years) were assigned to the control group and 17 (11 females; $24.63 \pm 0.14$ years) to the baseline group. For Experiment 2, 24 individuals were separated into two groups. Before performing the experimental task, 12 participants received virtual lesioning of DLPFC (5 females; $24.04 \pm 0.23$ years) and the other 12 received virtual lesioning of pSTS (9 females; $22.54 \pm 0.18$ years). The Edinburgh Handedness Questionnaire (Oldfield, 1971) revealed that all participants were strongly right-handed (>90). All participants had normal or corrected-to-normal vision, were free of neurologic disorders, and had no motor impairments of the right upper limb. Participants gave written informed consent and were financially compensated for their time. The protocol was in accordance with the Declaration of Helsinki and was approved by the local ethical committee of kU Leuven, Belgium (Project s60072).

\section{Experimental setup}

Experimental task. Subject and actor were comfortably seated opposite to each other in front of a table (for the experimental setup, see Fig. 1A). Participants were required to grasp and lift the manipulandum (see Acquisition of force data) that was placed in front of them in turns with the actor. As such, one trial consisted of one lifting action performed by either the actor (actor trial) or the participant (participant trial). Before the start of the task, participants received two practice trials on the objects with a congruent size-weight relationship (congruent objects) but not on those with an incongruent relationship (incongruent objects; for an explanation, see Acquisition of force data). Participants also received the following instructions beforehand: (1) Lift the manipulandum to a height of $\sim 5 \mathrm{~cm}$ at a smooth pace that is natural to you. (2) Only place thumb and index finger on the graspable surfaces (precision grip). (3) The cube in your trial always matches the cube in the actor's preceding trial both in size and weight. As such, participants always lifted the exact same cube as the actor did in the preceding trial and could rely on lift observation to estimate object weight for their own trials (Rens and Davare, 2019). Finally, both participants and actor were asked to place their hand on a predetermined location on their side of the table to ensure consistent reaching throughout the experiment. Reaching distance was $\sim 25 \mathrm{~cm}$ and required participant and actor to use their entire right upper limb to reach for the manipulandum. Last, participants were not informed about the incongruent objects before the start of the experiment.

For Experiment 1 (control and baseline groups), each trial performed by the actor or the participant was initiated with a neutral sound cue (start cue). For Experiment 2 (DLPFC and pSTS groups), we removed the start cue as we applied TMS during participant trials as well (for the stimulation conditions, see TMS procedure and EMG recording; for the intergroup differences, see Experimental groups). Accordingly, participants in Experiment 2 were instructed to consider the TMS pulse as the start cue and only initiate their movement after TMS was applied. For all groups, trials lasted $4 \mathrm{~s}$ to ensure that participants and actor had enough time to reach, grasp, and lift the manipulandum smoothly at a natural pace. Intertrial interval was $\sim 5 \mathrm{~s}$ during which the cuboid in the manipulandum could be changed. A transparent switchable screen (Magic Glass), placed in front of the participant's face, became transparent at trial onset and turned back to opaque at the end of the trial. The screen remained opaque during the intertrial interval to ensure participants had no vision on the cube switching. The actor always performed the act of changing the cuboid before executing his trials (even if the same cube would be used twice in a row). This was done to ensure that participants could not rely on sound cues to predict cube weight in the actor's 
A

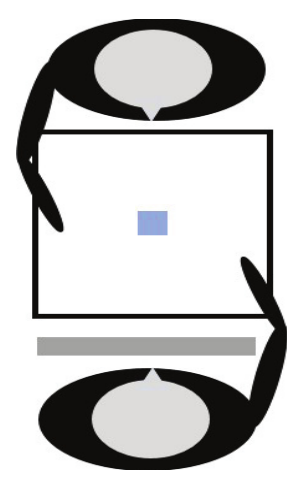

B

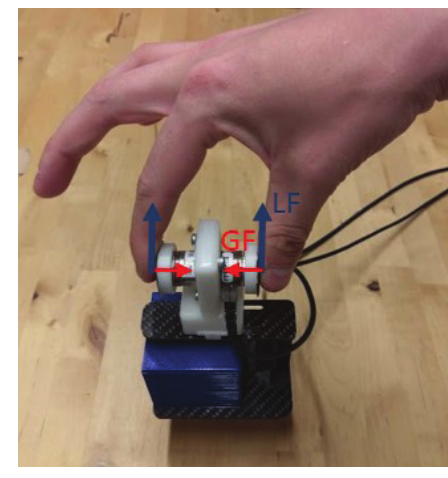

C
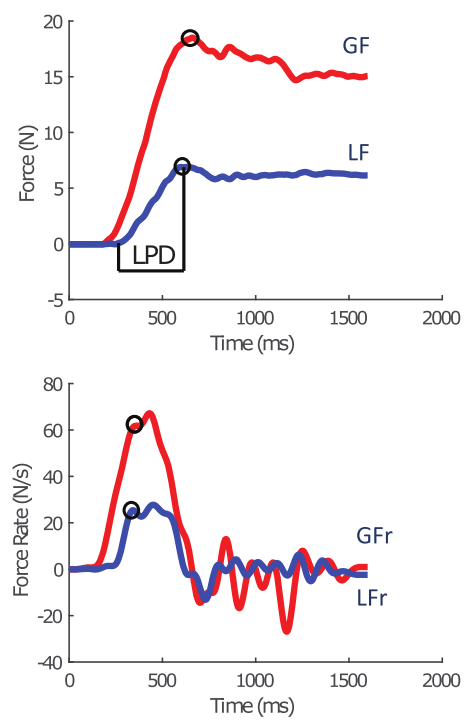

Figure 1. Experimental setup. $\boldsymbol{A}$, Representation of the experimental setup: the participant and actor were seated opposite to each other in front of a table on which the manipulandum was positioned. A switchable screen was placed in front of the participant's face. $\boldsymbol{B}$, Photograph of the grip-lift manipulandum used in the experiment. LF (blue) and GF (red) vectors are indicated. C, GF and LF typical traces (top) and their derivatives (bottom) for a skilled lift. Circles represent first peak values used as parameters. LPD was defined as the delay between object contact (GF $>0.20 \mathrm{M}$ ) and object lift-off (LF $>0.98 \times$ object weight). Figure reprinted with permission from Rens and Davare (2019).

Table 1. Distribution of trials per group ${ }^{a}$

\begin{tabular}{|c|c|c|c|c|c|c|c|c|c|}
\hline \multirow[b]{2}{*}{ Group } & \multirow[b]{2}{*}{ TMS applied } & \multicolumn{3}{|c|}{$\begin{array}{l}\text { Amount of observed } \\
\text { congruent lifts }\end{array}$} & \multicolumn{3}{|c|}{$\begin{array}{l}\text { Amount of observed } \\
\text { incongruent lifts }\end{array}$} & \multirow[b]{2}{*}{$\begin{array}{l}\text { Total amount of } \\
\text { observed lifts }\end{array}$} & \multirow[b]{2}{*}{$\begin{array}{l}\text { Ratio of } \\
\text { incongruent trials }\end{array}$} \\
\hline & & None & $\begin{array}{l}\text { At observed } \\
\text { contact }\end{array}$ & $\begin{array}{l}\text { After observed } \\
\text { lift-off }\end{array}$ & None & $\begin{array}{l}\text { At observed } \\
\text { contact }\end{array}$ & $\begin{array}{l}\text { After observed } \\
\text { lift-off }\end{array}$ & & \\
\hline Control & & 12 & 36 & 36 & 0 & 0 & 0 & 84 & - \\
\hline Baseline & & 32 & 32 & 32 & 0 & 24 & 24 & 144 & $33 \%$ \\
\hline DLPFC & & 24 & 0 & 48 & 0 & 0 & 24 & 96 & $25 \%$ \\
\hline
\end{tabular}

${ }^{a}$ For each group, the amount of observed trials for each TMS condition is presented. Amount of observed congruent lifts: Half the amount of observed congruent lifts consisted of the big-heavy object and the other half of the small-light object. Amount of observed incongruent lifts: Half the amount of observed incongruent lifts consisted of the big-light object and the other half of the small-heavy object. Participants lifted the objects themselves after lift observation. As such, the total amount of trials (observation and execution) is double the amount of the "observed trials."

upcoming trial. Switching actions were never performed before participant trials as they were explained that their cube would always match that of the actor.

Experimental procedure. All participants performed the object-lifting task in a single session (experimental session). Moreover, participants of Experiment 2 underwent prior MRI scanning (session duration: $30 \mathrm{~min}$ ) on a different day. At the start of the experimental session (start of scanning session for the participants of Experiment 2), participants gave written informed consent and were prepared for TMS stimulations as described below. Afterward, participants performed the experimental task (for the amount of trials per group, see Table 1). Experimental sessions lasted $60 \mathrm{~min}$ for the control group and $90 \mathrm{~min}$ for the baseline, DLPFC, and pSTS groups. Differences in session duration between the groups resulted from differences in TMS preparation and the amount of trials per group (see below).

Experimental groups. In Experiment 1, we wanted to investigate whether the presence of incongruent objects alters motor resonance. To do so, we divided participants into two groups: the control and baseline group. Participants in the control group were only exposed to the congruent objects. In contrast, participants in the baseline group lifted both the congruent and incongruent objects during the task.

In Experiment 2, we wanted to investigate how pSTS and DLPFC are causally involved in mediating the suppressive mechanism revealed in Experiment 1. Participants performed the same task as the baseline group of Experiment 1 (i.e., interacting with both the congruent and incongruent objects) after receiving a virtual lesion over either pSTS or DLPFC.
Trial amount per experimental group. First, we initially considered the incongruent objects to be pivotal for investigating how motor resonance is driven by expected and actual object weight. We decided on 12 trials per object condition for the incongruent objects ( 12 for smallheavy and 12 for big-light; 24 in total for both incongruent objects combined) based on Senot et al. (2011). Their study is, to our knowledge, one of the few that investigated motor resonance during observation of "live" (no video recordings) observation of object lifting. As they found consistent results using 10 trials per condition, we decided to include two more due to our experimental task. These two extra trials were intended to serve as a buffer for potential errors made by the actor or the participants.

Second, we decided to use unequal proportions of congruent and incongruent objects based on Alaerts et al. (2012). They demonstrated that, during lift observation, the observer's motor system predictively encodes object weight during the observed reaching phase. However, it is important to note that they used a blocked design, enabling participants to anticipate object weight, although the objects were visually identical. Considering that we did not want to rely on a blocked design but a pseudo-randomized one, we argued that unequal proportions would cause participants to expect that size was indicative of weight, causing motor resonance to be driven by these size-driven weight expectations at observed contact. In contrast, we argued that, if presented with equal proportions, participants would entirely ignore the size cue (as it could indicate either weight) eradicating motor resonance at observed object contact. 
Third, we initially wanted $25 \%$ of trials to be incongruent for all groups interacting with both congruent and incongruent objects. However, this was not feasible for the baseline group as this would cause their behavioral task to last twice as long compared with the other groups. Accordingly, we increased the amount of incongruent trials to $33 \%$ for the baseline group. This proportion was selected based on Pavone et al. (2016). They showed that neural activity, recorded with EEG, is different when observing correctly ( $70 \%$ of trials) and incorrectly ( $30 \%$ of trials) executed grasping actions in virtual reality. Importantly, this proportional difference between our baseline group (33\%) on one side and the DLPFC and pSTS groups (25\%) on the other side should not have affected motor resonance differently: Pezzetta et al. (2018) demonstrated, using EEG, that observed errors rather than their probability elicit typical error-related cortical activation. Last, for all groups, the amount of congruent trials was defined with the intent of maintaining these proportions of incongruent trials.

Fourth, as our findings for the baseline group showed that motor resonance was not modulated at observed contact, we decided to remove this TMS timing condition for the DLPFC and pSTS groups. This was done to ensure that the behavioral task was completed before the disruptive effects of cTBS, lasting $\sim 1$ h (Huang et al., 2005), ran out.

Fifth, to investigate whether TMS during lift observation did not interfere with the participants' lift planning, we included a non-TMS condition for the congruent objects (33\% of congruent trials amount).

Last, we included the control experiment due to our unanticipated findings in the baseline group. As our baseline group findings showed that TMS did not interfere with predictive lift planning, we decided to reduce the amount of non-TMS trials. We decided to include two more trials (18 in total) for each (congruent) condition compared with the baseline experiment. These trials were intended to serve as a buffer for potential errors made by the actor or the participant and to ensure we minimally had 16 correct congruent trials.

Object lifting sequences. A unique pseudo-randomized object lifting sequence was generated for each participant of each group using a custom-written MATLAB script. For the baseline group, this sequence was divided over four experimental blocks. For participants in the control, DLPFC, and pSTS groups, this sequence was divided over two experimental blocks. Participants received a short break between experimental blocks. Pseudo-randomization was based on the following criteria: (1) Within each experimental block, objects of the same condition were presented an equal amount of times (e.g., in a given experimental block, half the amount of congruent objects were big-heavy, whereas the other half was small-light). (2) Each object for each TMS timing was presented an equal amount of times in each experimental block (e.g., for the baseline group, lift observation of the big-heavy object when TMS was applied at observed object contact was presented 4 times in each of the four experimental blocks). (3) Each experimental block of the baseline, DLPFC, and pSTS groups could not start with an incongruent trial. (4) Two incongruent trials were separated by at least one congruent trial. (5) Half the amount of incongruent trials, in a given experimental block, were performed in the first half of that experimental block and the other half of incongruent trials in the second half of that experimental block.

\section{Acquisition of force data}

A grip-lift manipulandum consisting of two 3D force-torque sensors was attached to a custom-made carbon fiber basket in which different objects could be placed (for an image of the manipulandum, see Fig. 1B). The total weight of the manipulandum was $1.2 \mathrm{~N}$. The graspable surface (17 $\mathrm{mm}$ diameter and $45 \mathrm{~mm}$ apart) of the force sensors was covered with fine sandpaper (P600) to increase friction. For the present experiment, we used four 3D-printed objects. The large objects (cuboids) were $5 \times$ $5 \times 10 \mathrm{~cm}$ in size, whereas the two small ones (cubes) measured $5 \times 5 \times$ $5 \mathrm{~cm}$. Two of the objects (one small and one large) were filled with lead particles so that each of them weighed $0.3 \mathrm{~N}$. The other two were filled with lead particles until each of them weighed $5 \mathrm{~N}$. Combined with the weight of the manipulandum, the light and heavy objects weighed 1.5 and $6.3 \mathrm{~N}$, respectively. Importantly, using these four objects, we had a $2 \times 2$ design with size (small or big) and weight (light or heavy) as factors. In addition, this design allowed us to have two objects that were congruent in size and weight (large objects are expected to be heavier than smaller ones of the same material) and two incongruent objects for which this size-weight relationship was inversed (Baugh et al., 2012). To exclude any visual cues indicating potential differences between the same-sized objects, they were hidden under the same paper covers. In the present study, we used two ATI Nano17 F/T sensors (ATI Industrial Automation). Both F/T sensors were connected to the same NI-USB 6221 OEM board (National Instruments), which was connected to a personal computer. Force data were acquired at $1000 \mathrm{~Hz}$ using a customwritten Labview script (National Instruments). Last, one of the authors (G.R.) served as the actor in both Experiments 1 and 2.

\section{TMS procedure and EMG recording}

General procedure. For all groups, EMG recordings were performed using $\mathrm{Ag}-\mathrm{AgCl}$ electrodes, which were placed in a typical belly-tendon montage over the right first dorsal interosseous muscle. A ground electrode was placed over the processus styloideus ulnae. Electrodes were connected to a NL824 AC preamplifier (Digitimer) and a NL820A isolation amplifier (Digitimer), which in turn was connected to a microl40-3 CED (Cambridge Electronic Design). EMG recordings were amplified with a gain of $1000 \mathrm{~Hz}$, high-pass filtered with a frequency of $3 \mathrm{~Hz}$, sampled at $3000 \mathrm{~Hz}$ using Signal software (Cambridge Electronic Design), and stored for offline analysis. For TMS stimulation, we used a DuoMAG 70BF coil connected to a DuoMAG XT-100 system (DEYMED Diagnostic). For M1 stimulation, the coil was tangentially placed over the optimal position of the head (hotspot) to induce a posterior-anterior current flow and to elicit motor-evoked potentials (MEPs) in right first dorsal interosseous muscle. The hotspot was marked on the scalp of each participant. Stimulation intensity ( $1 \mathrm{mV}$ threshold) for each participant was defined as the lowest stimulation intensity that produced MEPs $>1 \mathrm{mV}$ in at least 4 of 8 consecutive trials when stimulating at the predetermined hotspot. Last, the control group and baseline group received 12 stimulations at the $1 \mathrm{mV}$ threshold before and after the experiment to have a baseline measure of resting CSE. Moreover, for the baseline group, we also recorded a baseline measure of resting CSE halfway through the experiment (i.e., when participants had performed half of the experimental blocks) as their experimental session lasted 30 min longer.

Stimulation during the experimental task. For the control and baseline group, single-pulse TMS over M1, for probing CSE, was applied during the actor trials at two different timings: at observed object contact and $300 \mathrm{~ms}$ after observed object lift-off (for definitions of object contact and lift-off, see Data processing). Participants did not receive stimulations during their trials (i.e., participant trials).

For the DLPFC and pSTS groups, single-pulse TMS, over M1 for probing CSE, was applied during both the actor and participant trials. During observation, we only applied single-pulse TMS during the observed lifting phase, and not at observed contact for two reasons: (1) The results from Experiment 1 indicated that CSE was primarily modulated after observed object lift-off and (2) because of the time constraints related to the duration of the after-effects caused by cTBS (Huang et al., 2005), which are limited to $\sim 1 \mathrm{~h}$. During participant trials, single-pulse TMS was applied $400 \pm 100 \mathrm{~ms}$ (jitter) after object presentation. As participants were instructed to only start lifting after receiving the stimulation, it was applied during movement planning and not execution. We did not stimulate the control and baseline groups during lift planning because, initially, we were only interested in motor resonance. We then included these stimulations in Experiment 2 because we wanted to investigate the effect of a virtual lesion of DLPFC or pSTS on CSE modulation during motor planning and whether these effects would be different from those during action observation. Finally, in Experiment 1 (control and baseline groups), we did not use neuro-navigation but relied on the hotspot mark on the scalp to apply single-pulse TMS over M1 during the experiment. In contrast, for Experiment 2 (DPLFC and pSTS groups), we used neuro-navigation for applying cTBS over these regions but also for maintaining the same coil positioning and orientation when applying single-pulse TMS over M1 during the experiment. Accordingly, for Experiment 2, the hotspot was determined using the same procedures as in Experiment 1, although the single-pulse TMS stimulations over M1 during the experiment were neuro-navigated. 
However, this should not have affected the validity of our between-group differences (e.g., see: Jung et al., 2010).

Additional procedures for Experiment 2. After defining the $1 \mathrm{mV}$ threshold, we defined the active motor threshold (aMT) as the lowest stimulation intensity that produced MEPs that were clearly distinguishable from background EMG during a voluntary contraction of $\sim 20 \%$ of their maximum using visual feedback. Before the experimental task, participants received cTBS over either DLPFC or pSTS. cTBS consisted of bursts of three pulses at $50 \mathrm{~Hz}$, repeated with a frequency of $5 \mathrm{~Hz}$ and at an intensity of $80 \%$ of the aMT for $40 \mathrm{~s}$ (600 pulses in total). It has been considered that this type of repetitive stimulation disrupts activity within the stimulation region for a period up to $60 \mathrm{~min}$ (Huang et al., 2005). Consequently, it has often been termed a "virtual lesion." In Experiment 2, we also collected resting CSE before cTBS. As such, we recorded three resting CSE measurements, that is, pre-cTBS, pretask ( 5 min after cTBS ended and just before the start of the experimental task), and post-task. To ensure that cTBS was applied on the desired stimulation area, a highresolution structural T1-weighted anatomic image of each participant was acquired with an MPRAGE sequence (Philips Ingenia 3.0T CX, TR/ $\mathrm{TE}=9.72 / 4.60 \mathrm{~ms} ;$ voxel size $=1.00 \times 1.00 \times 1.00 \mathrm{~mm}^{3} ; \quad \mathrm{FOV}=256 \times$ $256 \times 192 \mathrm{~mm}^{3}$; 192 coronal slices), which was coregistered during the experiment with the fiducial landmarks using a Brainsight TMS neuronavigation system (Rogue Research).

DLPFC was anatomically identified following Mylius et al. (2013). Briefly, we identified the superior and inferior frontal sulci as the superior and inferior borders of the middle frontal gyrus. The posterior border was defined as the precentral sulcus and the frontal one as the anterior termination of the olfactory sulcus in the coronal plane. Last, the middle frontal gyrus was divided equally into three parts and the separating line between the anterior and middle thirds was defined as the DLPFC (for full details, see Mylius et al., 2013). We always defined DLPFC within the middle frontal sulcus. This allowed us to consistently target the middle frontal sulcus using the same coil orientation across participants. Coil orientation was perpendicular to the middle frontal sulcus with the handle pointing downward. pSTS was anatomically defined following Cattaneo et al. (2010) and Arfeller et al. (2013) as the middle between the caudal and rostral ends of the ascending branch of STS, just below the intraparietal sulcus. Coil orientation was perpendicular to pSTS with the handle pointing downward. The mean \pm SEM Talairach coordinates for these sites were as follows: left DLPFC: $x=$ $-38.14 \pm 0.93, y=23.53 \pm 1.64, z=32.29 \pm 0.80$; left pSTS: $x=-54.03 \pm$ $1.09, y=-49.86 \pm 1.32, z=9.35 \pm 1.22$ as estimated on the cortical surface (for stimulation locations, see Fig. 2), which are in line with previous studies [left DLPFC: $x=-42.17 \pm 5.07, y=-33.73 \pm 5.73, z=32.36 \pm$ 6.17, Mylius et al. (2013); left pSTS: $x=-51.6 \pm 3.6, y=-43.2 \pm 7.1$, $z=7.1 \pm 6.4$, Arfeller et al. (2013)].

\section{Data processing}

Force data. Data collected with the F/T sensors were low-pass filtered with a fifth-order Butterworth filter (forces cutoff frequency: $30 \mathrm{~Hz}$; force rates cutoff frequency: $15 \mathrm{~Hz}$ ). A custom script was written in MATLAB to compute the following variables: (1) grip force (GF) and load force (LF), which were defined as the exerted force perpendicular and tangential to the normal force, respectively (Fig. 1B). GF and LF were computed as the sum of the respective force components exerted on both sensors. Additionally, GF rate (GFr) and LF rate (LFr) were computed by taking the first derivative of GF and LF, respectively. We report not GF and LF but their rates (Fig. 1C), as it has been demonstrated that force rate parameters are a reliable indicator of predictive force scaling (Johansson and Westling, 1988; Gordon et al., 1991). For analyses purposes of the force parameters, we decided to use the first peak grip and LFr values after object contact that were at least $30 \%$ of the maximum peak rate. This threshold was used to exclude small peaks in the force rates due to noise or small bumps caused by lightly contacting the F/T sensors. In addition, we decided to use the first peak force rate values as later peak values might be contaminated with feedback mechanisms and not reflect predictive force planning (Castiello, 2005; Rens and Davare, 2019). Accordingly, using the peak force rates enabled us to investigate whether participants scaled their fingertips forces differently for the incongruent

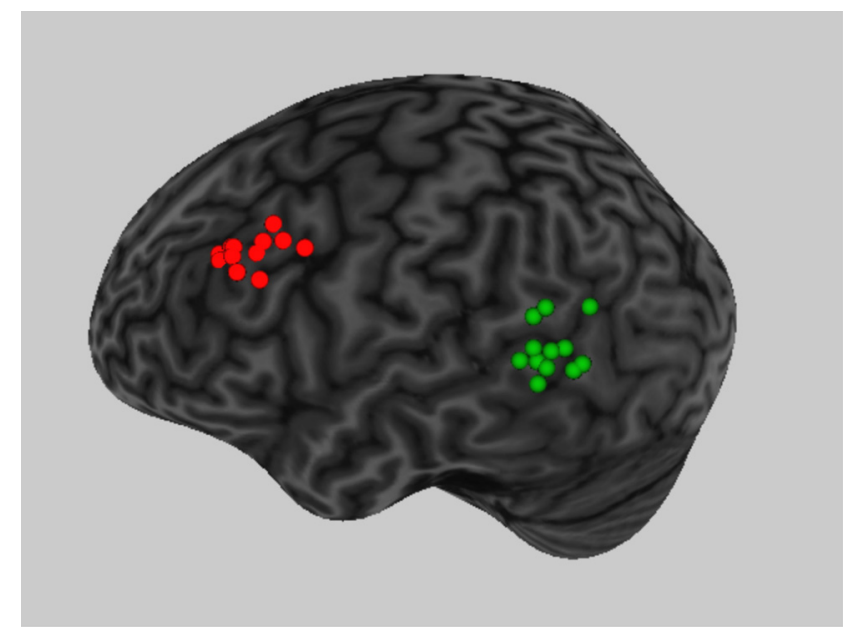

Figure 2. Stimulation sites. Anatomical locations where cTBS was applied for each individual subject of the DLPFC (red) and pSTS (green) groups.

and congruent objects. In addition to peak force rates, we also report the loading phase duration (LPD), which was defined as the latency between object contact and lift-off. Object contact and lift-off were defined as the time points when GF exceeded $0.2 \mathrm{~N}$ and LF exceeded $0.98 \times$ object weight (Fig. 1C), respectively (these definitions were used for timing the TMS stimulation during lift observation; see: TMS procedure and EMG recording). In addition, GF and LF were required to stay above these thresholds for at least $200 \mathrm{~ms}$. We included LPD as it is considered an estimator of the lifting speed (e.g., the shorter the LPD the faster the object will be lifted) (Johansson and Westling, 1988), which is a movement parameter used by participants to estimate object weight (Hamilton et al., 2007). Moreover, we could also use this parameter to investigate the participants' lifting performance. Last, both force rate parameters and LPD were $z$-score normalized. For the participants, $z$-score normalization was done for each participant separately. For the actor, $z$-score normalization was also done for each "participant" separately. That is, the actor's lifting performance in one session (as observed by 1 participant) was $z$-score normalized against the data of only that session. We decided to normalize our data based on the assumption that the actor's lifting speed might vary and this might affect the participants' lifting speed as well. Accordingly, $z$-score normalization would enable us compare between-group differences.

EMG data. From the EMG recordings, we extracted the peak-to-peak amplitudes of the MEP using a custom-written MATLAB script. All EMG recordings were visually inspected for background noise related to muscle contractions. Moreover, trials were excluded when the MEP was visibly contaminated (i.e., spikes in background EMG) or when an automated analysis found that the average background EMG was $>50 \mu \mathrm{V}$ (root-mean-square error) in a time window of $200 \mathrm{~ms}$ before the TMS stimulation. We also assessed prestimulation (background) EMG by calculating the root-mean-square error scores across a $100 \mathrm{~ms}$ interval ending $50 \mathrm{~ms}$ before TMS stimulation. Last, for each participant separately, we excluded outliers, which were defined as values exceeding the mean \pm 3 SDs. For each participant, all MEPs collected during the experimental task (but not resting measurements) were normalized with $z$ scores using their grand mean and SD. For Experiment 2, $z$-scoring was done for lift observation and planning separately.

\section{Statistical analysis}

CSE during rest. To investigate within-group differences in baseline CSE, we performed repeated-measures ANOVAs (ANOVA $A_{R M}$ ) for the control and the baseline group separately with one within-factor resting state (control: pretask and post-task; baseline; pretask, between experimental blocks, post-task). For Experiment 2, we performed a mixed ANOVA with between-factor group (DLPFC or pSTS) and within factor resting (pre-cTBS, pretask, post-task).

Within-group differences for CSE during the experimental task. First, to investigate whether our experimental task can elicit weight-driven 
motor resonance effects during lift observation, we performed an ANOVA $_{\mathrm{RM}}$ on the control group only with within-factors cube (big-heavy or small-light) and timing (observed contact or after observed lift-off). To investigate whether the presence of the incongruent objects altered motor resonance, we used a GLM (due to different effect sizes) to probe potential differences between the control and baseline groups on the congruent objects only. We used the between-factor group (control or baseline) and within-factors cube and timing. Because of our findings, we followed up on this GLM with an $\mathrm{ANOVA}_{\mathrm{RM}}$, only performed on the baseline group with within-factors timing, size (big or small), and weight (heavy or light).

After these analyses on the groups of the first experiment, we investigated the potential effects of the virtual lesions of DLPFC and pSTS. For this, we performed a GLM with between-factor group (baseline, DLPFC, or pSTS) and within-factors size and weight. As we did not stimulate the DLPFC and pSTS groups at observed contact, we could not include the within-factor timing. As we wanted to further explore potential withingroup effects, we followed up on the GLM with separate $\mathrm{ANOVA}_{\mathrm{RM}} \mathrm{s}$ for the DLPFC and pSTS groups with within-factors size and weight. Finally, to explore potential differences between lift observation and planning for the groups of Experiment 2, we performed a final GLM with between-factor group (DLPFC or pSTS) and within-factors action (observation or planning), size, and weight.

Within-group differences in background EMG during the experimental task. To ensure that differences in CSE during the behavioral task were not driven by between-condition variations in background EMG, we performed the analyses described in the preceding paragraph on the background EMG as well.

Force parameters of the participants. For each parameter of interest (peak GFr, peak LFr, and LPD), we performed a GLM on the congruent objects only with between-factor group (control, baseline, DLPFC, or pSTS) and within-factor cube (big-heavy or small-light). We performed an additional GLM on the congruent and incongruent objects combined with between-factor group (baseline, DLPFC, or pSTS; control not included due to not using the incongruent objects) and within-factors size and weight. Importantly, within-factors related to the timing of the TMS stimulation are not included here as our preliminary analyses indicated that it did not affect predictive force planning in the participants (i.e., we did not find significance for any of the relevant pairwise comparisons). Based on these findings, we decided to pool the data for timing and present the data as such for clarity.

Force parameters of the actor. For each parameter (peak GFr, peak LFr, and LPD), we performed the same analyses as described in Force parameters of the participants. We did not include the within-factors related to timing as the actor was blinded to the timings during the experiment.

Last, for the GLMs, we used Type III sum of squares; comparisons of interest exhibiting statistically significant differences $(p \leq 0.05)$ were further analyzed using the Holm-Bonferroni test. All data presented in the text are given as mean \pm SEM. All analyses were performed in Statistica (Dell).

\section{Results}

In the present study, we investigated how motor resonance is modulated during lift observation. For this, participants performed an object-lifting task in turns with an actor. The control group only lifted objects with a congruent size-weight relationship (i.e., big-heavy and small-light objects). The baseline group lifted objects with both congruent and incongruent size-weight relationships (i.e., additional big-light and small-heavy objects). The subject groups participating in Experiment 2 (DLPFC and pSTS groups) used the same objects as the baseline group. Importantly, they performed the experimental task after receiving a TMS induced virtual lesion over either DLPFC or pSTS. Only relevant main and interaction effects are reported below.

\section{Stimulation intensities}

To examine differences between stimulation intensities of the different groups, we ran two GLMs to investigate group differences in $1 \mathrm{mV}$ thresholds (all groups) and aMT (DLPFC and pSTS groups only). All values are expressed as a percentage of the maximal stimulator output. As expected, the GLM failed to reveal any significant difference between groups for the $1 \mathrm{mV}$ stimulation intensity (control $=61 \pm 2.62 \%$; baseline $=55.64 \pm$ $3.26 \%$; DLPFC $=57.54 \pm 3.26 \%$; pSTS $=50.46 \pm 3.00 \% ; F_{(3,48)}=$ $\left.2.39 p=0.08, \eta_{\mathrm{p}}^{2}=0.13\right)$ as well as for the aMT (DLPFC $=$ $42.82 \pm 2.26 \% ; \mathrm{pSTS}=38.46 \pm 2.08 \% ; F_{(1,22)}=2.01 p=0.17$, $\left.\eta_{\mathrm{p}}^{2}=0.08\right)$. Note that the degrees of freedom of the error are lower due to missing values.

We informally asked participants in Experiment 2 how they perceived cTBS. In the DLPFC group, 2 of 12 participants described cTBS as "uncomfortable," whereas the other 10 did not report negative sensations. In the pSTS group, 5 participants reported negative sensations: 4 reported the sensations as "uncomfortable" and 1 as "painful." Last, no one reported other physical adverse effects (e.g., dizziness or headaches) that could potentially have been related to the single-pulse or cTBS stimulations.

\section{CSE at rest}

Experiment 1. For the control (pretask $=0.89 \pm 0.08 \mathrm{mV}$; posttask $=1.16 \pm 0.22 \mathrm{mV}$ ) and baseline groups (preblock $1=0.61 \pm$ $0.06 \mathrm{mV}$; between-blocks $=0.79 \pm 0.18 \mathrm{mV}$; post-block $2=0.87 \pm$ $0.17 \mathrm{mV}$ ), both analyses provide no evidence that resting CSE changed significantly over time (nonsignificance of timing; both $F<1.67$, both $p>0.21$, both $\eta_{\mathrm{p}}^{2}<0.09$ ).

Experiment 2. Both the main effects of group, timing as well as their interaction effect, were not significant (all $p>0.16$ ), providing no evidence that resting CSE differed between groups or changed over time (DLPFC: pre-cTBS $=1.16 \pm 0.26 \mathrm{mV}$, pretask $=$ $1.53 \pm 0.22 \mathrm{mV}$, post-task $=1.60 \pm 0.44 \mathrm{mV}$; pSTS: pre-CTBS $=$ $2.04 \pm 0.26 \mathrm{mV}$, pretask $=1.60 \pm 0.22 \mathrm{mV}$, post-task $=2.20 \pm 0.44$ $\mathrm{mV})$.

\section{Background EMG during the experiment}

To ensure that between-group and between-condition differences were not driven by differences in hand relaxation during lift observation and planning, we investigated potential differences in background EMG. For this, we used the same statistics as described in Statistical analyses (Within-group differences for CSE during the experimental task). Briefly, all main and interaction effects across all analyses, except for one, were not significant (all $F<1.99$, all $p>0.18$, all $\eta_{\mathrm{p}}^{2}<0.11$ ). The interaction effect action (observe or plan lift) $\times$ size $($ small or big $) \times$ group (DLPFC or pSTS) was significant $\left(F=5.14, p=0.03, \eta_{\mathrm{p}}^{2}=0.19\right)$. However, the post hoc analysis failed to reveal significant differences between any of the conditions. These findings provide no evidence that background EMG different significantly between and within groups.

\section{CSE during the experimental task}

With the control group, we investigated whether our task can elicit weight-driven modulation of CSE during observed object lifting. As shown in Figure 3, the analysis substantiated the validity of our setup: When the control group observed lifts of the big-heavy object (big-heavy $=0.07 \pm 0.03$ ) CSE was significantly facilitated compared with when they observed lifts of the smalllight object (small-light $=-0.08 \pm 0.03 ; p=0.02$; main effect of cube: $\left.F_{(1,17)}=6.87, p=0.02, \eta_{\mathrm{p}}^{2}=0.29\right)$.

Afterward, we explored whether the presence of the incongruent objects affected motor resonance. For this, we compared the control and baseline groups for only the congruent objects. In line with our findings for the control group, CSE was 

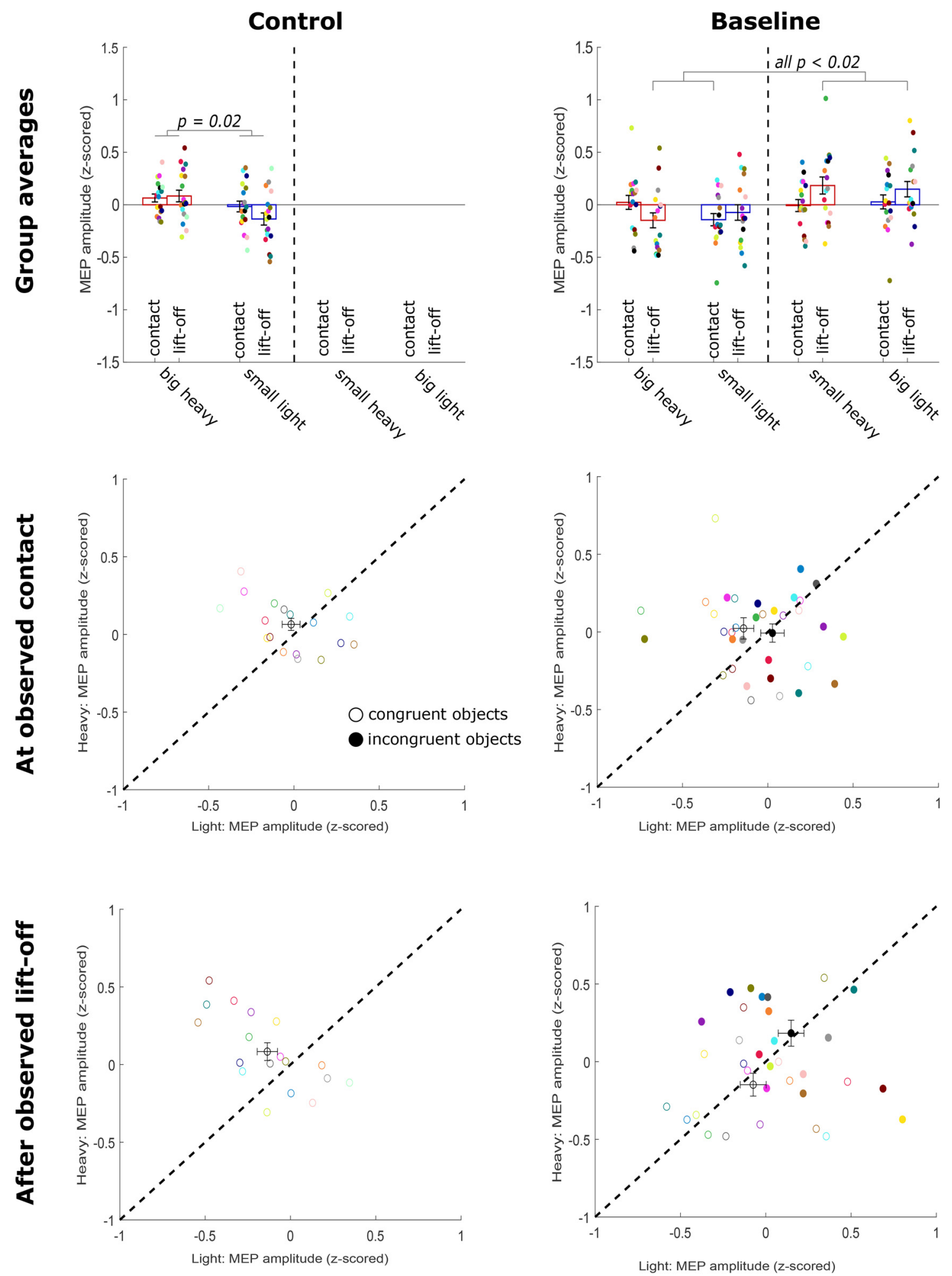

Figure 3. Modulation of CSE during lift observation in the control and baseline group. Top row, Average MEP values (z-scored) during lift observation pooled across participants for the control (left) and baseline group (right). Left and right of the dashed line on each figure represent the congruent (big-heavy and small-light) and incongruent (small-heavy and big-light) objects, respectively. Red represents heavy weights. Blue represents light weights. For the control and baseline groups, we used two TMS timings during observation (i.e., at observed object contact and after observed lift-off). As such, of two adjacent bars, the first and second one represent MEP values at observed contact and during observed lifting respectively. Middle and bottom rows, $2 D$ visualization of the average MEP values (z-scored) at observed object contact (middle row) and after observed lift-off (bottom row). MEP values for the heavy and light objects are shown on the $y$ and $x$ axis, respectively. Each participant within each group is represented by two same colored circles (scatter). Empty circles represent the congruent objects (MEP values for bigheavy on the $y$ axis and for small-light on the $x$ axis). Filled circles represent the incongruent objects (MEP values for small-heavy on the $y$ axis and for big-light on the $x$ axis). Black circles represent the group average \pm SEM for the respective conditions. Black dashed line indicates the equation $y=x$ and indicates the line of no CSE modulation. Accordingly, scatter circles above or below the dashed line represent that CSE, when observing lifts of heavier objects, was increased or decreased, respectively. No intragroup significant differences are shown on the middle and bottom rows. 
significantly facilitated when observing lifts of the big-heavy cube (big-heavy $=0.006 \pm 0.02$ ) compared with the small-light one (small-light $=-0.09 \pm 0.03 ; p=0.04$; main effect of cube: $\left.F_{(1,33)}=4.34, p=0.04, \eta_{\mathrm{p}}^{2}=0.12\right)$. However, the main effect of group $\left(F_{(1,33)}=7.30, p=0.01, \eta_{\mathrm{p}}^{2}=0.18\right)$ was significant as well: When observing lifts (of the congruent objects), CSE of the baseline group (congruent objects $=-0.09 \pm 0.02$ ) was significantly more inhibited than that of the control group (congruent objects $=0.00 \pm 0.02$ ). Considering that the group averages for CSE (MEP amplitude) are calculated using $z$-score normalization, these findings indicate that the presence of the incongruent objects in the baseline experiment should have inhibited CSE modulation for the congruent objects (due to negative $z$ score). In addition, the interaction effect cube $\times$ timing $\times$ group $\left(F_{(1,33)}\right.$ $\left.=3.71, p=0.06, \eta_{\mathrm{p}}^{2}=0.10\right)$ was borderline significant. Because of this borderline significance, we decided to explore how the presence of the incongruent objects in the baseline group affected modulation of motor resonance.

To further probe potential differences between the congruent and incongruent objects for the baseline group, we performed a separate $\mathrm{ANOVA}_{\mathrm{RM}}$ on the baseline group with within-factors timing, size, and weight. Interestingly, this analysis revealed that CSE modulation in the baseline group was not driven by size or weight but by "congruency." As shown in Figure 3, CSE was significantly more facilitated for the small-heavy object during observed lifting (mean $=0.18 \pm 0.08)$ compared with the bigheavy one during observed lifting (mean $=-0.15 \pm 0.07$; $p=0.01)$ and the small-light one at observed contact (mean = $-0.14 \pm 0.06 ; p=0.02$ ) (interaction effect of weight $\times$ size $\times$ timing: $\left.F_{(1,16)}=7.54, p=0.01, \eta_{\mathrm{p}}^{2}=0.32\right)$. Conversely, CSE was significantly more facilitated during observed lifting of the big-light object (mean $=0.15 \pm 0.08$ ), compared with the big-heavy one during observed lifting $(p=0.03)$, and the small-light one at observed contact $(p=0.04)($ size $\times$ weight $\times$ timing). Importantly, these findings contradict our initial hypothesis: We expected that motor resonance would be driven by size at observed contact and afterward by weight during observed lifting. However, our results demonstrated that motor resonance effects driven by size or weight were "masked" by a mechanism that is monitoring object congruency (i.e., monitoring a potential mismatch between anticipated and actual object weight).

With the pSTS and DLPFC groups, we investigated the potential effects of the virtual lesions on CSE modulation during lift observation. As described in Statistical analysis, we performed a GLM with between-factor group (baseline, DLPFC, and pSTS groups) and within-factors size and weight. As shown in Figure 4 , this analysis revealed that, for the pSTS group, CSE was significantly facilitated when observing lifts of heavy objects, regardless of their size (heavy objects $=0.11 \pm 0.05$ ) compared with lifts of the light ones (light objects $=-0.12 \pm 0.04 ; p=0.03$ ) (interaction effect of group $\times$ weight: $\left.F_{(2,38)}=4.97, p=0.01, \eta_{\mathrm{p}}^{2}=0.17\right)$. However, this weight-driven modulation of CSE during lift observation was absent for the baseline group (due to the congruency effect as described above; heavy objects $=0.02 \pm 0.04$; light objects $=0.04 \pm 0.03 ; p=1.00)$ but was also absent for the DLPFC group (heavy objects $=-0.02 \pm 0.05$; light objects $=$ $0.02 \pm 0.04 ; p=1.00)$ (group $\times$ weight). As such, these findings indicate that weight-driven modulation of CSE during lift observation was restored for the pSTS group. However, these results do not provide any evidence that CSE was modulated after virtually lesioning DLPFC.
To further investigate the weight effect in the pSTS group, we performed an additional GLM for the control and pSTS groups combined. Indeed, if weight-driven modulation of CSE during lift observation was restored by virtual lesioning of pSTS, then the pSTS group should have not differed significantly from the control group with respect to the congruent objects. For this analysis, we used the between-factor group (control and pSTS) and within-factor cube (big-heavy and small-light) for timing being only after observed lift-off (as we did not apply TMS at observed contact in the pSTS group). Importantly, the main effect of cube was significant $\left(F_{(1,28)}=6.43, p=0.02, \eta_{\mathrm{p}}^{2}=0.19\right)$. In line with our control group findings, CSE was significantly facilitated when observing lifts of the big-heavy object (bigheavy $=0.08 \pm 0.04)$ compared with observing lifts of the light one (small-light $=-0.09 \pm 0.04 ; p=0.01$ ). Interestingly, this analysis did not show significance for the main effect of group as well as for its interaction with cube (both $F<0.03$, both $p>0.28$, both $\left.\eta_{\mathrm{p}}^{2}<0.04\right)$. As such, these findings further substantiate that, in both the control and pSTS group, CSE modulation during lift observation was driven by the object's actual weight (Figs. 3, 4).

Moreover, we explored whether CSE was still modulated by object weight after virtual lesioning of DLPFC using the same analysis as described in the preceding paragraph [GLM with between-factor group (control and DLPFC) and within-factor cube (big-heavy and small-light)]. Briefly, this analysis failed to reveal significance for any of the main effects (group and cube; both $F<0.84$, both $p>0.37$, both $\eta_{\mathrm{p}}^{2}<0.03$ ) as well as their interaction effect $\left(F=3.57, p=0.06\right.$, all $\left.\eta_{\mathrm{p}}^{2}=0.11\right)$. It is important to note that, in the first paragraph of this Results section (CSE during the experimental task), we already demonstrated for the control group that CSE modulation during lift observation was driven by object weight. Accordingly, considering that the interaction effect group $\times$ cube was borderline significant and that the DLPFC group is included in this analysis, we decided to perform a final ANOVA $A_{R M}$ on the DLPFC group only with one within-factor cube (big-heavy and small-light). This was done to investigate whether CSE modulation in the DLPFC group was driven by cube. This analysis failed to show significance for cube $\left(F_{(1,11)}=0.54, p=0.48, \eta_{\mathrm{p}}^{2}=0.05\right)$. In conclusion, these analyses provide no evidence at all that CSE was modulated during lift observation when DLPFC was virtually lesioned.

To end, we investigated whether CSE was modulated differently during lift observation and planning for the DLPFC and pSTS groups using a GLM with between-factor group and within-factors action (observation or planning), size, and weight. Interestingly, this analysis showed that CSE was significantly facilitated when observing or planning lifts of the heavy objects (heavy objects $=0.03 \pm 0.02$ ) compared with the light ones (light objects $=-0.05 \pm 0.02 ; p=0.02$; main effect of weight: $F_{(1,22)}=$ $\left.6.68, p=0.02, \eta_{\mathrm{p}}^{2}=0.23\right)$. However, this weight effect was likely driven by the pSTS group as the significant interaction effect group $\times$ weight $\left(F_{(1,22)}=5.66, p=0.03, \eta_{\mathrm{p}}^{2}=0.20\right)$ revealed that weight drove CSE modulation in the pSTS (heavy objects= $0.06 \pm 0.02$; light objects $=-0.08 \pm 0.03 ; p=0.01$ ) but not in the DLPFC group (heavy objects $=-0.00 \pm 0.02$; light objects $=$ $-0.01 \pm 0.03 ; p=1.00)$. In its turn, the significant difference between CSE modulation by the heavy and light objects for the pSTS group (group $\times$ weight) was likely driven by the triple interaction effect group $\times$ action $\times$ weight $\left(F_{(1,22)}=4.31\right.$, $\left.p=0.05, \eta_{\mathrm{p}}^{2}=0.16\right)$. Post hoc exploration of this significant interaction effect revealed that, for the pSTS group, CSE was significantly facilitated during lift observation of the heavy objects 

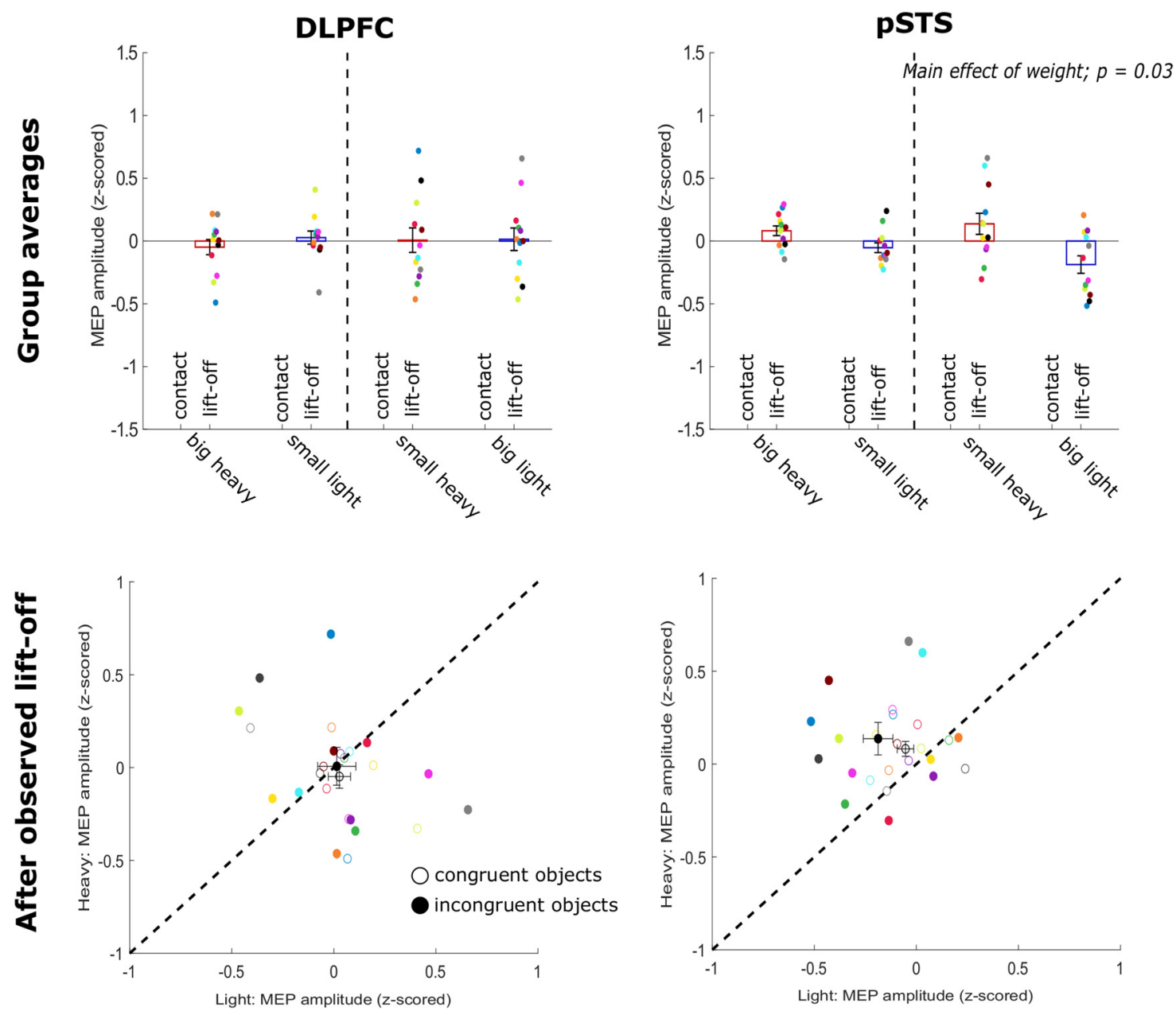

Figure 4. Modulation of CSE during lift observation in the DLPFC and pSTS groups. Top row, Average MEP values (z-scored) during lift observation pooled across participants for the DLPFC (left) and PSTS group (right). Left and right of the dashed line on each figure represent the congruent (big-heavy and small-light) and incongruent (small-heavy and big-light) objects, respectively. Red represents heavy weights. Blue represents light weights. Bottom row, 2D visualization of the average MEP values (z-scored) after observed lift-off. MEP values for the heavy and light objects are shown on the $y$ and $x$ axis, respectively. Each participant within each group is represented by two same colored circles (scatter). Empty circles represent the congruent objects (MEP values for big-heavy on the $y$ axis and for small-light on the $x$ axis). Filled circles represent the incongruent objects (MEP values for small-heavy on the $y$ axis and for big-light on the $x$ axis). Black circles represent the group average \pm SEM for the respective conditions. Black dashed line indicates the equation $y=x$ and indicates the line of no CSE modulation. Accordingly, scatter circles above or below the dashed line represent that CSE, when observing lifts of heavier objects, was increased or decreased, respectively. No intragroup significant differences are shown on the bottom row.

(heavy objects $=0.11 \pm-0.03$ ) compared with of the light ones (light objects $=-0.12 \pm 0.03 ; p=0.04$ ), whereas this difference was absent during planning (heavy objects $=$ $0.02 \pm 0.04$; light objects $=-0.04 \pm 0.04 ; p=1.00$ ). In conclusion, these findings provide no evidence that CSE was modulated in the pSTS and DLPFC groups during lift planning (Fig. 5). As we have no "control conditions" (group without virtual lesioning during lift planning), these findings cannot be further interpreted.

To sum up, our results demonstrate that, when participants only interact with objects having a congruent sizeweight relationship (i.e., big-heavy or small-light), CSE during lift observation is modulated by the object weight as indicated by the size and/or the movement kinematics (control group). Interestingly, when objects with incongruent sizeweight relationship (i.e., big-light and small-heavy) were included (baseline group), weight-driven modulation of CSE was "suppressed" and CSE was modulated by "object congruency" instead. That is, CSE was facilitated during observed lifting of objects with incongruent properties compared with objects with congruent properties.
Moreover, our results also highlighted that virtual lesioning of PSTS abolishes the suppressive mechanism monitoring the observer's weight expectations and restores weightdriven modulation of CSE during lift observation. As such, our results provide evidence for the causal involvement of pSTS in modulating CSE by monitoring the observer's weight expectations during the observation of hand-object interactions. In addition, virtual lesioning of DLPFC eradicated both the suppressive mechanism as well as weightdriven motor resonance: During lift observation, we found no evidence that CSE was modulated at all. Accordingly, these findings suggest that DLPFC is causally involved in a "general" modulation of CSE during the observation of hand-object interactions. To end, we did not find significant differences between the DLPFC and pSTS groups for lift planning. Considering that we have no "control" group with which to compare, these findings cannot be further interpreted.

\section{Force parameters of the participants}

As mentioned before, we pooled all data with respect to factors related to TMS timing as preliminary analyses revealed that 

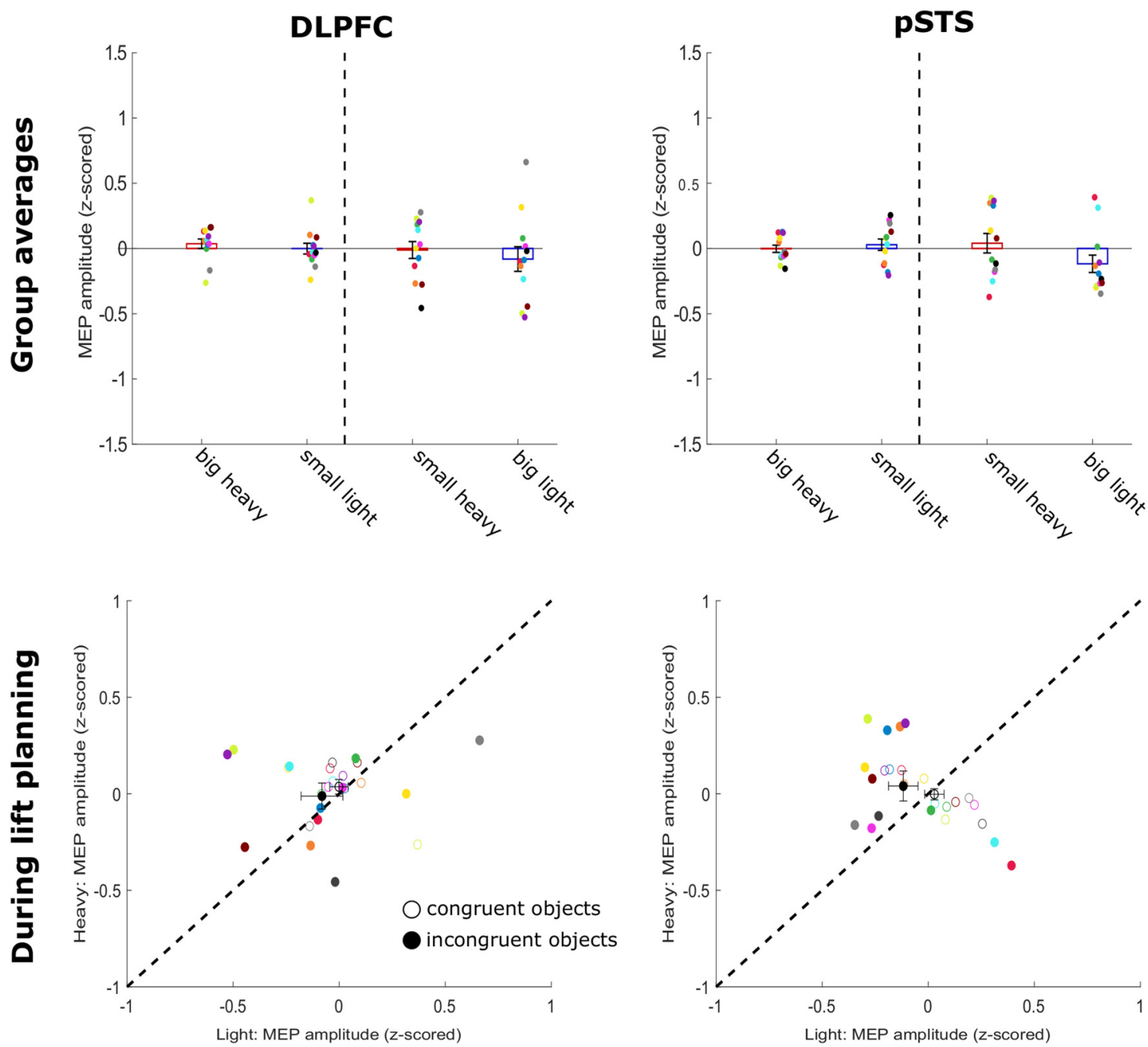

Figure 5. Modulation of CSE during lift planning in the DLPFC and pSTS groups. Top row, Average MEP values (z-scored) during lift planning pooled across participants for the DLPFC (left) and pSTS group (right). Left and right of the dashed line on each figure represent the congruent (big-heavy and small-light) and incongruent (small-heavy and big-light) objects, respectively. Red represents heavy weights. Blue represents light weights. Bottom row, 2D visualization of the average MEP values (z-scored) during lift planning. MEP values for the heavy and light objects are shown on the $y$ and $x$ axis, respectively. Each participant within each group is represented by two same colored circles (scatter). Empty circles represent the congruent objects (MEP values for big-heavy on the $y$ axis and for small-light on the $x$ axis). Filled circles represent the incongruent objects (MEP values for small-heavy on the $y$ axis and for big-light on the $x$ axis). Black circles represent the group average \pm SEM for the respective conditions. Black dashed line indicates the equation $y=x$ and indicates the line of no CSE modulation. Accordingly, scatter circles above or below the dashed line represent that CSE, when planning lifts of heavier objects, was increased or decreased, respectively. No intragroup significant differences are shown on the bottom row.

predictive force planning of the participants was not altered by single-pulse TMS.

\section{Normalized peak GFrs}

For both the group comparisons on the congruent objects only (all four groups) and on the objects with both congruency types (baseline, DLPFC, and pSTS groups), neither the main effect of group nor any of its interactions effects were significant (all $F<0.86$, all $p>0.47$, all $\eta_{\mathrm{p}}^{2}<0.04$ ).

First, for only the congruent objects, these findings suggest that there is no evidence that the experimental groups scaled their GFs (i.e., peak GFr values) differently, regardless of whether they were exposed to only congruent object (control group) or to both congruent and incongruent objects (baseline, DLPFC, and pSTS groups). Second, these findings also provide no evidence that virtual lesioning of either DLPFC or pSTS (DLPFC and pSTS groups) affected predictive GF scaling based on lift observation compared with receiving no virtual lesioning (control and baseline groups). Aside from these results, all groups increased their GFs significantly faster for the big-heavy cube (bigheavy $=0.48 \pm 0.03)$ than for the small-light one (small-light $=$ $-0.43 \pm 0.03$; main effect of cube: $\left(F_{(1,55)}=353.70, p<0.001\right.$, $\left.\eta_{\mathrm{p}}^{2}=0.87\right)$. All group averages are shown in Figure 6 .

Moreover, these findings are similar for the groups that interacted with both congruent and incongruent objects. That is, the baseline, DLPFC, and pSTS groups increased their GFs significantly faster for the heavy objects (heavy $=0.38 \pm 0.03$ ) than for the light ones (light $=-0.39 \pm 0.02 ; p<0.001$; main effect of weight: $\left.F_{(1,38)}=255.93, p<0.001, \eta_{\mathrm{p}}^{2}=0.87\right)$. However, although these groups were able to scale their GFs to the actual object weight, they were still biased by the size as they increased their GFs significantly faster for the big objects (big objects= $0.08 \pm 0.02$ ) than for the smaller ones (small objects $=-0.10 \pm$ $0.02 ; p<0.001$; main effect of size: $F_{(1,38)}=23.69, p<0.001$, $\left.\eta_{\mathrm{p}}^{2}=0.38\right)$. Last, post hoc analysis of the significant interaction effect weight $\times$ size $\left(F_{(1,38)}=5.42, p=0.025, \eta_{\mathrm{p}}^{2}=0.12\right)$ highlighted that these groups also increased their GFs significantly faster for the big-heavy object (big-heavy $=0.50 \pm 0.03$ ) than for 

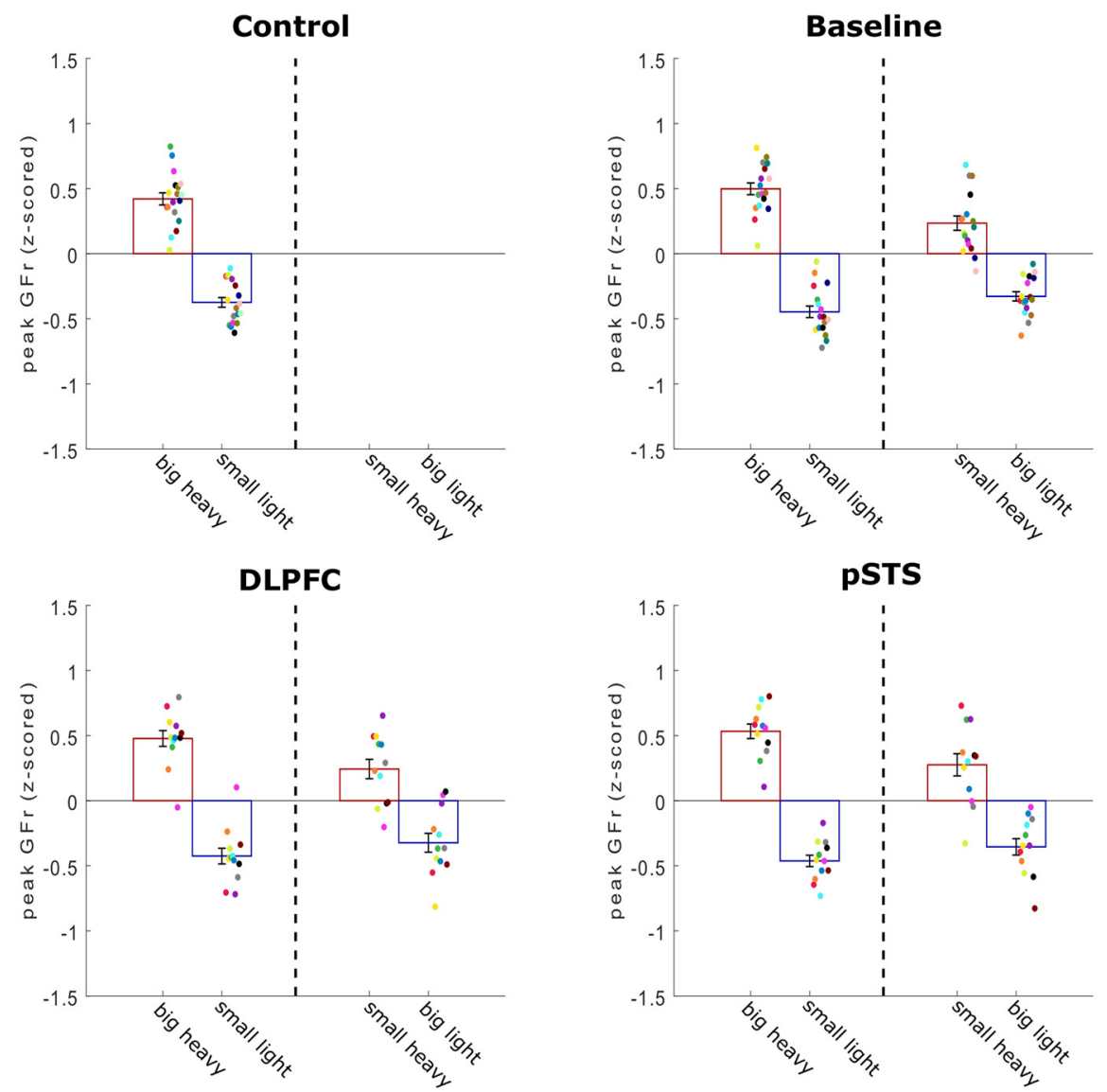

Figure 6. Peak GFrs of the participants. Average peak GFr value (z-scored) for each group separately. Left and right of the dashed line on each figure represent the congruent (big-heavy and small-light) and incongruent (small-heavy and big-light) objects, respectively. Within each experimental group, each colored circle (scatter) represents the average peak GFr value for 1 participant in that specific condition. Data are mean \pm SEM. No intragroup significant differences are shown.

the small-heavy one (small-light $=0.25 \pm 0.04 ; p<0.001$ ). This difference was absent for the light objects (small-light $=$ $-0.44 \pm 0.03$; big-light $=-0.34 \pm 0.03 ; p=0.08$ ).

\section{Normalized peak LFrs}

The findings for peak LFr were nearly identical to those for peak GFr. Indeed, for both comparisons (congruent objects only: all groups; both congruent and incongruent objects: baseline, DLPFC, and pSTS groups), the main effect of group as well as all its interaction effects were not significant (all $F<0.72$, all $p>0.49$, all $\left.\eta_{\mathrm{p}}^{2}<0.04\right)$. Accordingly, we did not find any evidence that predictive LF planning based on lift observation was affected by (1) the presence of the incongruent objects (control group vs baseline, DLPFC, and pSTS groups) or by (2) the virtual lesioning of DLPFC or pSTS (control and baseline groups vs DLPFC and pSTS groups). Similar to our findings for peak GFr, participants increased their LFs significantly faster for the bigheavy cube (big-heavy $=0.42 \pm 0.02$ ) than for the small-light one (small-light $=-0.39 \pm 0.02 ; p<0.001 ;$ main effect of cube: $\left(F_{(1,55)}=339.57, p<0.001, \eta_{\mathrm{p}}^{2}=0.86\right)$.

Again, the baseline, DLPFC, and pSTS groups, which interacted with both congruent and incongruent objects, increased their LFs significantly faster for the heavy objects (heavy= $0.35 \pm 0.02$ ) than for the light ones (light $=-0.35 \pm 0.2$; $p<0.001$; main effect of weight: $F_{(1,38)}=304.80, p<0.001, \eta_{\mathrm{p}}^{2}=$
0.89), although they were also biased by object size (big: peak $\mathrm{LFr}=0.05 \pm 0.02$; small: peak $\mathrm{LFr}=-0.05 \pm 0.02 ; p=0.004$; main effect of size: $F_{(1,38)}=9.10, p=0.005$, $\left.\eta_{\mathrm{p}}^{2}=0.19\right)$. All group averages are shown in Figure 7 without intragroup significant differences being shown.

\section{Normalized LPD}

Our findings for the participants' LPD were identical to those for peak GFr: For congruent objects only (all groups) and the congruent and incongruent objects combined (baseline, DLPFC, and pSTS groups), our analyses did not show significance for the main effect of group as well as its interaction effects (all $F<2.07$, all $p>0.140$, all $\eta_{\mathrm{p}}^{2}<0.10$ ), again suggesting that our experimental groups did not differ significantly from each other. Again, the GLM for the congruent objects only showed that the main effect of cube was significant $\left(F_{(1,55)}=2717.64, p<0.001\right.$, $\left.\eta_{\mathrm{p}}^{2}=0.90\right)$, indicating that all groups lifted the big-heavy object (big-heavy $=0.83 \pm$ 0.02) slower than the small-light one ( small-light $=-0.80 \pm 0.02 ;<0.001)$.

In line with our peak GFr findings, the groups (baseline, DLPFC, and pSTS) interacting with both congruent and incongruent objects lifted the heavy objects (heavy= $0.91 \pm 0.03)$ significantly slower than the light ones (light $=-0.80 \pm 0.02 ; p<0.001$; main effect of weight: $F_{(1,38)}=1139.85$, $\left.p<0.001, \eta_{\mathrm{p}}^{2}=0.97\right)$, although they were still biased by the object size as they lifted the big objects faster than the small ones (big $=0.01 \pm 0.01 ;$ small $=0.09 \pm 0.02 ; p<$ 0.001 ; main effect of size: $F_{(1,38)}=18.43$, $\left.p<0.001, \eta_{\mathrm{p}}^{2}=0.33\right)$. Finally, post hoc analysis of the significant interaction effect weight $\times$ size $\left(F_{(1,38)}=23.33, p<0.001, \eta_{\mathrm{p}}^{2}=\right.$ 0.38 ) revealed that all groups lifted the big-heavy object (bigheavy $=0.82 \pm 0.02)$ significantly faster than the small-heavy one (small-heavy $=0.99 \pm 0.04 ; p<0.001$ ), although this difference was absent for the light objects (small-light $=-0.81 \pm 0.02$; biglight $=-0.80 \pm 0.03 ; p=1.00$ ). All group averages are shown in Figure 8 without intragroup significant differences being shown.

To sum up, participants lifted the objects (size: big or small by weight: heavy or light) in turns with the actor and were instructed that the object in their trial was always identical, both in terms of size and weight, to the object the actor lifted in the previous trial. As such, participants could potentially rely on lift observation to estimate object weight and plan their own lifts accordingly. Importantly, our results support this notion: In line with Rens and Davare (2019), our results demonstrate that the groups who interacted with both the congruent and incongruent objects were able to detect the incongruent objects based on observed lifts performed by the actor. Indeed, our findings for the baseline, DLPFC, and pSTS groups showed that subjects scaled their fingertip forces to the actual weight of the incongruent objects (main effect of weight). However, it is important to note that these groups were still biased by object size as, on average, subjects scaled their fingertip forces faster for the large 
objects than for the small ones (main effect of size). Moreover, exploration of the significant interaction effect of weight $\times$ size for peak GFr and LPD indicated that this effect was primarily driven by the significant difference between heavy objects. Last, considering that we did not find significant differences between the baseline group on one side and the DLPFC and pSTS groups on the other side shows that virtual lesioning of either region did not affect predictive lift planning based on lift observation. As such, our findings related to the force parameters indicate that DLPFC and pSTS are not causally involved in either weight perception during lift observation or in updating the motor command based on lift observation.

\section{Force parameters of the actor Normalized peak GFrs}

Comparing the congruent objects only across all four groups, the actor increased his GFs significantly faster for the big-heavy object (big-heavy $=0.8 \pm 0.02$ ) than for the small-light one (small-light $=-0.79 \pm 0.01$; $p<0.001$; main effect of weight: $F_{(1,55)}=$ 3328, $\left.p<0.001, \eta_{\mathrm{p}}^{2}=0.98\right)$. Although the main effect of group was not significant, the interaction effect of group $\times$ cube $\left(F_{(3,55)}=\right.$ 5.85, $\left.p=0.002, \eta_{\mathrm{p}}^{2}=0.24\right)$ was. Post hoc analysis of this interaction effect showed that the actor scaled his GFs significantly faster for the big-heavy object in the baseline group (baseline: big-heavy $=0.89 \pm 0.03$ ) compared with the control group (control: big-heavy $=0.76 \pm 0.03, p=0.02$ ). However, all other between-group differences in the actor's lifting performance for the big-heavy object were not significant (DLPFC: bigheavy $=0.88 \pm 0.04$; pSTS: big-heavy $=0.78 \pm 0.03$; all $p>0.12$ ). Conversely, this was identical for the small-light object with the actor scaling his GFs significantly slower for the small-light object in the baseline group (baseline: small-heavy $=-0.84 \pm 0.02$ ) than in the control group (control: small-heavy $=-0.72 \pm 0.02 ; p=$ 0.05). Again, all other between-group actor differences for the small-light object were not significant (DLPFC: small-light = $-0.83 \pm 0.03$; pSTS: small-light $=-0.76 \pm 0.03$; all $p>0.24$ ).

For the comparisons including the incongruent objects (baseline, DLPFC, and PSTS groups), it is important to note that the interaction effect size $\times$ weight $\left(F_{(1,38)}=5.52, p=0.02, \eta_{\mathrm{p}}^{2}=\right.$ $0.13)$ was significant. Post hoc analysis showed that the actor increased his GFs similarly for the light objects (small-light = $-0.81 \pm 0.02$; big-light $=-0.83 \pm 0.03 ; p=1.00$ ) but not for the heavy ones (big-heavy $=0.85 \pm 0.02$; small-heavy $=0.79 \pm 0.04$; $p=0.03$ ). As our results indicate that the actor increased his GFs slower for the small-heavy object compared with the big-heavy object, suggesting that he was biased by the object's size during his own trials.

\section{Normalized peak LFrs}

In line with our findings for GFrs, the actor increased his LFs significantly faster for the big-heavy cuboid (big-heavy $=0.80 \pm$ 0.02 ) than the small-light one (small-light $=-0.72 \pm 0.02$;

\section{Control}
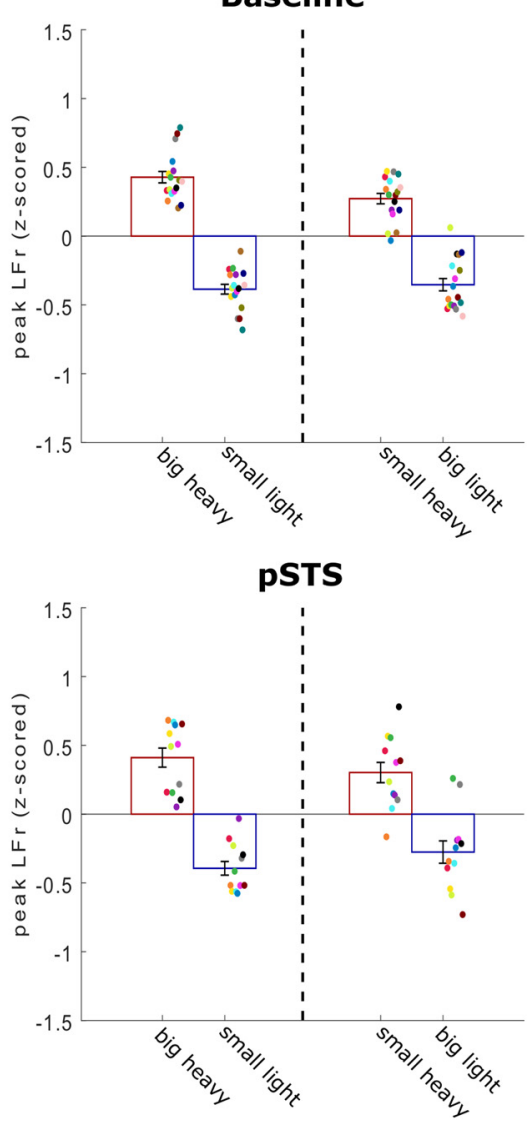

Figure 7. Peak LFrs of the participants. Average peak LFr values (z-scored) for each group separately. Left and right of the dashed line on each figure represent the congruent (big-heavy and small-light) and incongruent (small-heavy and big-light) objects, respectively. Within each experimental group, each colored circle (scatter) represents the average peak LFr value for 1 participant in that specific condition. Data are mean \pm SEM. No intragroup significant differences are shown.

$p<0.001$ ) (congruent objects only: main effect of cube: $F_{(1,55)}=$ 1950.87, $\left.p<0.001, \eta_{\mathrm{p}}^{2}=0.97\right)$. Importantly, post hoc exploration of the significant interaction effect group $\times$ cube $\left(F_{(3,55)}=3.87\right.$, $\left.p=0.01, \eta_{\mathrm{p}}^{2}=0.17\right)$ did not reveal any relevant significant differences in the actor's performance between groups on the bigheavy object (control $=0.71 \pm 0.04$; baseline $=0.84 \pm 0.04$; DLPFC $=$ $0.85 \pm 0.04$; $\mathrm{pSTS}=0.79 \pm 0.04$; all $p>0.18$ ) or the small-light one (control $=-0.63 \pm 0.03$; baseline $=-0.76 \pm 0.03 ;$ DLPFC $=$ $-0.76 \pm 0.04$; pSTS $=-0.71 \pm 0.04$; all $p>0.18$ ).

However, the analysis on both the congruent and incongruent objects showed that the actor scaled his LFs differently based on object size for both the light objects (small-light $=-0.74 \pm 0.02$; big-light $=-0.82 \pm 0.03 ; p=0.05$ ) and the heavy ones (bigheavy $=0.83 \pm 0.03$; small-heavy $=0.74 \pm 0.04 ; p=0.04)($ size $\times$ weight: $\left.F_{(1,38)}=15.40, p<0.001, \eta_{\mathrm{p}}^{2}=0.29\right)$. Finally, it is important to note that neither the main effect of group nor its interaction effects were significant (all $F<1.03$, all $p>0.37$, all $\eta_{\mathrm{p}}^{2}<$ $0.5)$. As such, we did not find evidence that the actor scaled his LFs differently for the different experimental groups.

\section{Normalized LPD}

Comparing only the congruent objects across all four groups showed that LPD of the actor was significantly longer when lifting the big-heavy object (big-heavy $=0.76 \pm 0.02$ ) than the small-light one (small-light $=-0.85 \pm 0.02 ; p<0.001$ ) (congruent objects only: main effect of cube: $F_{(1,55)}=2883.95, p<0.001$, $\left.\eta_{\mathrm{p}}^{2}=0.98\right)$. For the comparison on both the congruent and 


\section{Control}

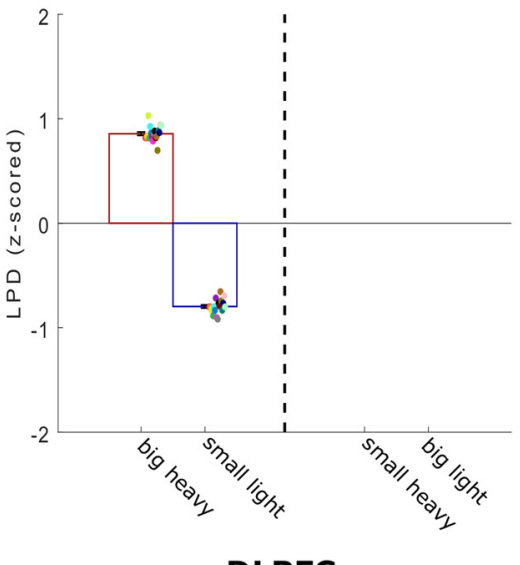

DLPFC

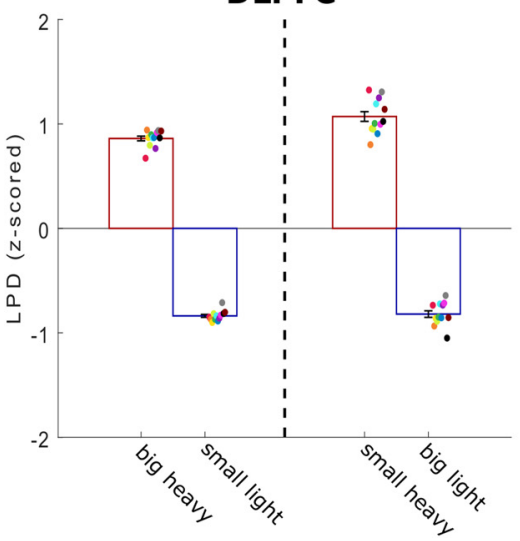

Baseline
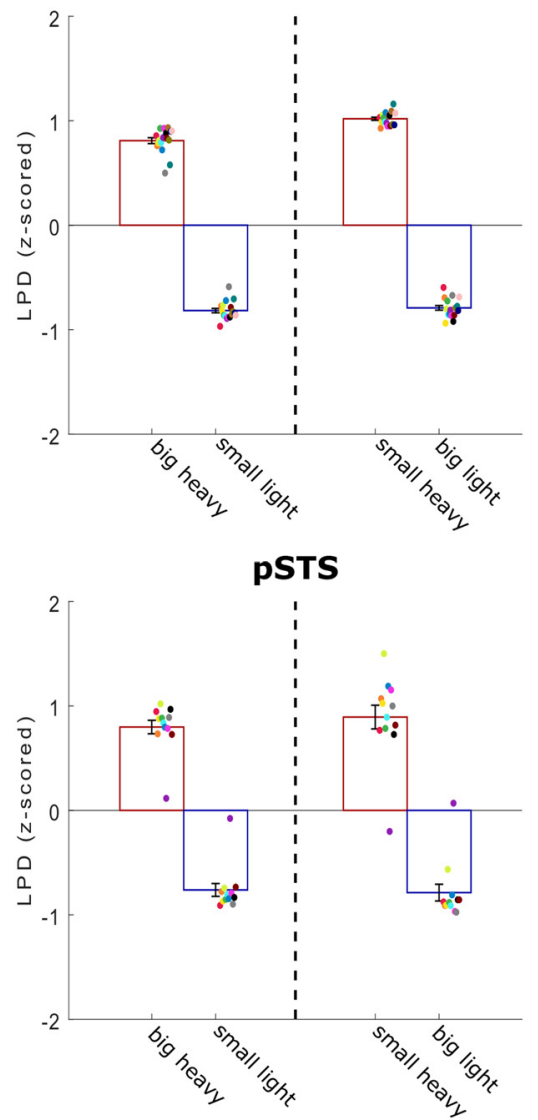

Figure 8. LPD of the participants. Average LPD values (z-scored) for each group separately. Left and right of the dashed line on each figure represent the congruent (big-heavy and small-light) and incongruent (small-heavy and big-light) objects, respectively. Within each experimental group, each colored circle (scatter) represents the average peak LPD value for 1 participant in that specific condition. Data are mean \pm SEM. No intragroup significant differences are shown.

incongruent objects, the interaction effect size $\times$ weight: $F_{(1,38)}=$ 57.40, $\left.p<0.001, \eta_{\mathrm{p}}^{2}=0.60\right)$ was significant. Critically, the post hoc analysis revealed that the actor lifted the small objects significantly slower than the big ones. That is, the LPD when lifting the big-heavy object (big-heavy $=0.76 \pm 0.02$ ) was significantly shorter than when lifting the small-heavy one (small-heavy= $0.89 \pm 0.03 ; p<0.001)$. Accordingly, this significant difference was also present for the light objects (small-light = $-0.84 \pm 0.02$; big-light $=-0.68 \pm 0.02 ; p<0.001)$. Although these findings suggest that the actor's lifting speed was biased by object size, he still lifted the light objects significantly faster than the heavy ones ( size $\times$ weight: all $p<0.001$ ).

In sum, these findings indicate that, in general, the actor scaled his fingertip forces toward the actual object weight for both the congruent and incongruent objects. However, it is important to note that the actor was biased by object size when interacting with the incongruent objects. Across all groups (except the control group which did not interact with the incongruent objects), the actor increased his fingertip forces faster for the big than for the small objects, resulting in a shorter LPD for the larger objects. Presumably, as participants were able to lift the objects (of which they could only predict object weight by relying on the actor's lifting) skillfully, it is plausible that these found differences in the actor's lifting performance drove the participants' ability to estimate object weight during observed lifting. Accordingly, these differences in observed lifting performance should also have driven modulation of CSE. Finally, except for one difference for normalized GFrs, the actor scaled his fingertip forces similarly across all groups. Importantly, these findings substantiate that our intergroup differences, with respect to CSE modulation, are not driven by differences in the actor's lifting performance between groups but rather by experimental setup differences (presence of incongruent vs only congruent objects; virtual lesioning of pSTS or vs no virtual lesion).

\section{Discussion}

First, we investigated how CSE is modulated during observation of lifting actions (i.e., "motor resonance"). Our control experiment findings align with previous literature (Alaerts et al., 2010a,b): When participants observed lifts of objects with a congruent only size-weight relationship, CSE was modulated by object weight. However, our baseline group findings highlight that weight-driven motor resonance effects are easily suppressed when weight cannot be reliably predicted based on size: When participants observed lifts of objects with congruent and incongruent size-weight relationships, CSE was larger when observing lifts of incongruent objects, regardless of their size and weight. Interestingly, this suggests that "typical" weightdriven motor resonance was suppressed by a mechanism monitoring size-weight congruence. However, we found these differences at different time points during action observation (Fig. 3), indicating that the baseline group perceived the small-light object weight before liftoff. Presumably, participants estimated weight based on the actor's reaching phase as Eastough and Edwards (2007) demonstrated that an individual's reaching phase depends on the object's mass. However, we cannot substantiate this assumption as we did not record the actor's reaching phase. Finally, in line with Rens and Davare (2019), the baseline group was able to generate the appropriate fingertip force scaling to lift the objects skillfully after lift observation.

Second, we investigated the causal involvement of top-down inputs in the suppressive mechanism monitoring size-weight congruence by disrupting either pSTS or DLPFC using cTBS. Strikingly, pSTS virtual lesions abolished the suppressive mechanism and restored weight-driven motor resonance, suggesting that pSTS is pivotal in monitoring weight expectations during lift observation. In contrast, DLPFC virtual lesions eradicated all modulation of motor resonance, suggesting that DLPFC is causally involved in the overall modulation of motor resonance. Although virtual lesions of DLPFC and pSTS altered motor resonance, we found no evidence that predictive lift planning, after lift observation, was affected. This suggests that adequate motor planning is not necessarily related to motor resonance effects.

Regarding our baseline group, Alaerts et al. (2010b) showed that, when participants observed lifts of objects with incongruent properties, motor resonance was still driven by weight as cued by 
the movement kinematics. Our results contrast theirs by showing that motor resonance was rather driven by size-weight congruence. Critically, our study differs from theirs on three major points: (1) participants in their study did not manipulate the objects; (2) their participants were not required to respond after observation (verbally or behaviorally); and (3) whereas we used a skewed proportion of congruent and incongruent trials, they used equal proportions.

It is unlikely that our baseline group findings are entirely driven by this skewed proportion of incongruent trials: Pezzetta et al. (2018) demonstrated using EEG that participants elicit typical error-monitoring activity whether larger or smaller proportions of erroneous grasping are observed. A plausible explanation, that our findings are driven by the experimental context rather than the skewed proportion, resides in another study of Alaerts et al. (2012): They demonstrated that motor resonance can reflect object weight predictively during observed reaching and, thus, when the actual object weight cannot yet be veridically identified. However, they used a blocked design and never challenged the participants' expectations. Thus, in our baseline group, randomly inserting trials with incongruent object size-weight properties might have caused a top-down mechanism to suppress weight-driven motor resonance. Arguably, this mechanism might be useful to prevent motor resonance from encoding object weight based on an incorrect prediction. That is, when a mismatch between expected and actual object weight is identified, this top-down mechanism releases all suppression allowing a sudden increase in CSE, which signals that the motor command will need to be updated from the one initially predicted based on object size, to the correct one based on the actor's lifting kinematics. As such, the contextual importance of accurately estimating object weight during observation might have driven this mechanism to suppress weight-driven motor resonance.

Motor resonance has been argued to rely on the putative human mirror neuron system (hMNS). First discovered in monkeys (di Pellegrino et al., 1992), mirror neurons are similarly activated when executing or observing the same action and have been argued to be involved in action understanding by "mapping" observed actions onto the cortical representations involved in their execution (Cattaneo and Rizzolatti, 2009). The hMNS is primarily located in M1, PMv, and API areas (Rizzolatti et al., 2014). Importantly, these regions also constitute the cortical grasping network which is pivotal in planning and executing grasping actions (for review, see Davare et al., 2011) further substantiating hMNS' involvement in action understanding.

However, Amoruso and Finisguerra (2019) argued that motor resonance only reflects an automatic replica of observed actions, if observed in isolation, but that it can be modulated by topdown inputs in presence of contextual cues. Our results support this hypothesis: Weight-driven motor resonance was present when weight expectations were never challenged (control group), but turned out to be suppressed when a size-weight mismatch was introduced (baseline group). Although we demonstrated a systematic effect of size-weight contingency on motor resonance, Figure 3 shows that the presence of incongruent trials (baseline group, right) also led to a larger between-subject variability compared with the control group (Fig. 3, left). This might be explained by the baseline group subjects relying on different strategies to extract weight-related information: either focusing on the movement kinematics or the size-weight contingency (Amoruso and Finisguerra, 2019).
In our second experiment, we investigated the origins of the suppressive mechanism and found that disrupting pSTS restores weight-driven motor resonance, suggesting that pSTS is causally involved in monitoring expectations during observation. These findings are plausible as pSTS is crucial in perceiving biological motion (Grossman et al., 2005), which is indicative of object weight (Hamilton et al., 2007), and in monitoring execution errors during observation (Pelphrey et al., 2004). Although pSTS does not contain mirror neurons (Hickok, 2009, 2013) and shares no connections with M1 (Iacoboni, 2005; Nelissen et al., 2011), it accesses the putative hMNS through reciprocal connections with AIP (Nelissen et al., 2011; Galletti and Fattori, 2018). Plausibly, pSTS modulates CSE through AIP-PMV and PMv-M1 connections (Davare et al., 2011; Gerbella et al., 2017). Indeed, our results suggest that pSTS monitors weight expectations during observed lifting and masks typical motor resonance effects when expectations can be incorrect. Plausibly, virtual lesioning of pSTS abolishes expectation-related input to AIP, restoring the automatic mapping of observed movement features. In addition, when expectations are never tested (control group), pSTS might not provide this top-down input and does not mask weightdriven motor resonance. However, future research is necessary to substantiate the latter.

We also investigated the causal involvement of DLPFC in monitoring weight expectations: Our results show that disrupting DLPFC eradicated both the expectation monitoring mechanism and also weight-driven motor resonance, arguing that DLPFC is pivotal in the overall modulation of CSE during lift observation, regardless of the underlying mechanism. Our results align with those of Ubaldi et al. (2015): They showed that, when motor resonance effects were altered by a visuomotor training task, the trained resonance could be eradicated by virtual lesioning of DLPFC, suggesting that DLPFC is critical in modulating rule-based motor resonance. Importantly, our results extend on theirs by demonstrating that virtual lesioning of DLPFC eradicates not only trained effects but also effects which are considered to be automatic. It is plausible that DLPFC can modulate motor resonance: Although DLPFC does not contain mirror neurons (Hickok, 2009, 2013), it is reciprocally connected with PMv (Badre and D'Esposito, 2009) and involved in action observation and processing contextual information (Raos and Savaki, 2017; Rozzi and Fogassi, 2017).

A limitation of the present study is that we used one TMS timing in the virtual lesion groups, due to time constraints. We only probed motor resonance after observed lift-off as we found the strongest effects of the suppressive mechanisms for our baseline group at this timing. In addition, Ubaldi et al. (2015) demonstrated that motor resonance driven by visuomotor associations is only altered during late, but not early, movement observation. Therefore, it seemed valid to focus on this timing. A second limitation concerns the absence of sham cTBS in Experiment 2. Noteworthy, virtual lesioning of DLPFC and pSTS modulated CSE differently, indicating that the stimulation site was relevant. However, probing motor resonance when observing lifts of congruent objects only, combined with cTBS disruption of DLPFC and pSTS, could further substantiate our findings. The last limitation is that we did not use a within-subject design. Considering our hypothesis that individuals' expectations alter motor resonance, we opted for a between-subject design to ensure all participants have the same expectations when performing the behavioral task (for the first time).

In conclusion, the present study shows that motor resonance is not robust but influenced by contextual differences. We argue 
that motor resonance should be carefully interpreted in light of the hMNS putative roles. Our results indicate that bottom-up motor resonance effects, driven by observed movement features, can only be probed when top-down suppressive expectation monitoring mechanisms from pSTS are not triggered. Moreover, DLPFC is pivotal in the global modulation of CSE during action observation. Together, these findings shed new light on the theoretical framework in which motor resonance effects occur and overlap with other cortical processing essential for the sensorimotor control of movements.

\section{References}

Alaerts K, Senot P, Swinnen SP, Craighero L, Wenderoth N, Fadiga L (2010a) Force requirements of observed object lifting are encoded by the observer's motor system: a TMS study. Eur J Neurosci 31:1144-1153.

Alaerts K, Swinnen SP, Wenderoth N (2010b) Observing how others lift light or heavy objects: which visual cues mediate the encoding of muscular force in the primary motor cortex? Neuropsychologia 48:2082-2090.

Alaerts K, de Beukelaar TT, Swinnen SP, Wenderoth N (2012) Observing how others lift light or heavy objects: time-dependent encoding of grip force in the primary motor cortex. Psychol Res 76:503-513.

Amoruso L, Finisguerra A (2019) Low or high-level motor coding? The role of stimulus complexity. Front Hum Neurosci 13:1-9.

Arfeller C, Schwarzbach J, Ubaldi S, Ferrari P, Barchiesi G, Cattaneo L (2013) Whole-brain haemodynamic after-effects of $1-\mathrm{Hz}$ magnetic stimulation of the posterior superior temporal cortex during action observation. Brain Topogr 26:278-291.

Badre D, D'Esposito M (2009) Is the rostro-caudal axis of the frontal lobe hierarchical? Nat Rev Neurosci 10:659-669.

Baugh LA, Kao M, Johansson RS, Flanagan JR (2012) Material evidence: interaction of well-learned priors and sensorimotor memory when lifting objects. J Neurophysiol 108:1262-1269.

Buckingham G, Wong JD, Tang M, Gribble PL, Goodale MA (2014) Observing object lifting errors modulates cortico-spinal excitability and improves object lifting performance. Cortex 50:115-124.

Castiello U (2005) The neuroscience of grasping. Nat Rev Neurosci 6:726736.

Cattaneo L, Rizzolatti G (2009) The mirror neuron system. Arch Neurol 66:557-560.

Cattaneo L, Sandrini M, Schwarzbach J (2010) State-dependent TMS reveals a hierarchical representation of observed acts in the temporal, parietal, and premotor cortices. Cereb Cortex 20:2252-2258.

Davare M, Kraskov A, Rothwell JC, Lemon RN (2011) Interactions between areas of the cortical grasping network. Curr Opin Neurobiol 21:565-570.

di Pellegrino G, Fadiga L, Fogassi L, Gallese V, Rizzolatti G (1992) Understanding motor events: a neurophysiological study. Exp Brain Res 91:176-180.

Eastough D, Edwards MG (2007) Movement kinematics in prehension are affected by grasping objects of different mass. Exp Brain Res 176:193198.

Fadiga L, Fogassi L, Pavesi G, Rizzolatti G (1995) Motor facilitation during action observation: a magnetic stimulation study. J Neurophysiol 73:2608-2611.

Galletti C, Fattori P (2018) The dorsal visual stream revisited: stable circuits or dynamic pathways? Cortex 98:203-215.

Gerbella M, Rozzi S, Rizzolatti G (2017) The extended object-grasping network. Exp Brain Res 235:2903-2916.

Gordon AM, Forssberg H, Johansson RS, Westling G (1991) Visual size cues in the programming of manipulative forces during precision grip. Exp Brain Res 83:447-482.

Grossman ED, Battelli L, Pascual-Leone A (2005) Repetitive TMS over posterior STS disrupts perception of biological motion. Vision Res 45:28472853.

Hamilton AC, Joyce DW, Flanagan JR, Frith CD, Wolpert DM (2007) Kinematic cues in perceptual weight judgement and their origins in box lifting. Psychol Res 71:13-21.
Hickok G (2009) Eight problems for the mirror neuron theory of action understanding in monkeys and humans. J Cogn Neurosci 21:1229-1243.

Hickok G (2013) Do mirror neurons subserve action understanding? Neurosci Lett 540:56-58.

Huang YZ, Edwards MJ, Rounis E, Bhatia KP, Rothwell JC (2005) Theta burst stimulation of the human motor cortex. Neuron 45:201-206.

Iacoboni M (2005) Neural mechanisms of imitation. Curr Opin Neurobiol 15:632-637.

Johansson RS, Westling G (1988) Coordinated isometric muscle commands adequately and erroneously programmed for the weight during lifting task with precision grip. Exp Brain Res 71:59-71.

Jung NH, Delvendahl I, Kuhnke NG, Hauschke D, Stolle S, Mall V (2010) Navigated transcranial magnetic stimulation does not decrease the variability of motor-evoked potentials. Brain Stimul 3:87-94.

Kilner JM (2011) More than one pathway to action understanding. Trends Cogn Sci 15:352-357.

Miller EK, Cohen JD (2001) An integrative theory of prefrontal cortex. Annu Rev Neurosci 24:167-202.

Mylius V, Ayache SS, Ahdab R, Farhat WH, Zouari HG, Belke M, Brugières P, Wehrmann E, Krakow K, Timmesfeld N, Schmidt S, Oertel WH, Knake S, Lefaucheur JP (2013) Definition of DLPFC and M1 according to anatomical landmarks for navigated brain stimulation: inter-rater reliability, accuracy, and influence of gender and age. Neuroimage 78:224232.

Nelissen K, Borra E, Gerbella M, Rozzi S, Luppino G, Vanduffel W, Rizzolatti G, Orban GA (2011) Action observation circuits in the macaque monkey cortex. J Neurosci 31:3743-3756.

Oldfield RC (1971) The assessment and analysis of handedness: the Edinburgh Inventory. Neuropsychologia 9:97-113.

Pavone EF, Tieri G, Rizza G, Tidoni E, Grisoni L, Aglioti SM (2016) Embodying others in immersive virtual reality: electro-cortical signatures of monitoring the errors in the actions of an avatar seen from a first-person perspective. J Neurosci 36:268-279.

Pazzaglia M, Smania N, Corato E, Aglioti SM (2008) Neural underpinnings of gesture discrimination in patients with limb apraxia. J Neurosci 28:3030-3041.

Pelphrey KA, Morris JP, McCarthy G (2004) Grasping the intentions of others: the perceived intentionality of an action influences activity in the superior temporal sulcus during social perception. J Cogn Neurosci 16:1706-1716

Pezzetta XR, Nicolardi V, Tidoni E, Aglioti SM (2018) Error, rather than its probability, elicits specific electrocortical signatures: a combined EEGimmersive virtual reality study of action observation. J Neurophysiol 120:1107-1118.

Raos V, Savaki HE (2017) The role of the prefrontal cortex in action perception. Cereb Cortex 27:4677-4690.

Rens G, Davare M (2019) Observation of both skilled and erroneous object lifting can improve predictive force scaling in the observer. Front Hum Neurosci 13:373.

Rizzolatti G, Cattaneo L, Fabbri-Destro M, Rozzi S (2014) Cortical mechanisms underlying the organization of goal-directed actions and mirror neuron-based action understanding. Physiol Rev 94:655-706.

Rossi S, Hallett M, Rossini PM, Pascual-Leone A (2011) Screening questionnaire before TMS: an update. Clin Neurophysiol 122:1686.

Rozzi S, FogassiL (2017) Neural coding for action execution and action observation in the prefrontal cortex and its role in the organization of socially driven behavior. Front Hum Neurosci 11:492.

Senot P, D'Ausilio A, Franca M, Caselli L, Craighero L, Fadiga L (2011) Effect of weight-related labels on corticospinal excitability during observation of grasping: a TMS study. Exp Brain Res 211:161-167.

Tidoni E, Borgomaneri S, di Pellegrino G, Avenanti A (2013) Action simulation plays a critical role in deceptive action recognition. J Neurosci 33:611-623.

Ubaldi SG, Barchiesi L Cattaneo (2015) Bottom-up and top-down visuomotor responses to action observation. Cereb Cortex 25:1032-1041. 